\title{
Inflammation and Ovarian Cancer
}

\author{
Antonio Macciò ${ }^{1}$ and Clelia Madeddu ${ }^{2}$ \\ ${ }^{1}$ Department of Obstetrics and Gynecology, Sirai Hospital, Carbonia, \\ ${ }^{2}$ Department of Medical Oncology, University of Cagliari, Cagliari \\ Italy
}

\section{Introduction}

Epithelial ovarian cancer (EOC) is a highly lethal gynaecological cancer for which overall prognosis has remained poor over the past few decades. A number of theories have been postulated in an effort to explain the aetiology of EOC. Noteworthy, these theories likely are not mutually exclusive, as they all converge more or less on the role of inflammation in promoting ovarian tumorigenesis and cancer progression. The tumour milieu in which ovarian carcinoma develops has been described as one enriched with a broad spectrum of pro-inflammatory cytokines and chemokines. In particular, several of these cytokines (such as tumour necrosis factor- $\alpha$ (TNF- $\alpha$ ), interleukin (IL)-1 $\beta$, and IL-6) produced by tumour itself or/and activated immune cells, besides stimulating cancer cell growth, have been shown to influence clinical disease status and prognosis, by reducing responsiveness to chemotherapy and inducing symptoms such as anorexia, altered energy metabolism, anaemia, weight loss, depression and fatigue. Recent data show that cytokine antagonists may have a role to play in the treatment of ovarian cancer. Their action by inhibiting both production and activity of inflammatory cytokines seems to obtain the control of angiogenetic and apoptotic events, the reversal of chemoresistance, the improvement of systemic symptoms and prognosis. In the light of our scientific research and the most recent experimental and clinical advances our chapter will review the most relevant and recent findings on the role of proinflammatory cytokines in the pathogenesis and prognosis of ovarian cancer and the possible therapeutic implications.

\section{Role of inflammation in the etiopathogenesis of ovarian cancer}

A number of studies suggest that factors related to inflammation of the ovarian surface epithelium (OSE), such as ovulation, endometriosis and pelvic inflammatory diseases, are associated with an increased risk for EOC. In particular, inflammatory mediators and several cytokines produced by activated innate immune cells, such as TNF- $\alpha$, IL-1 $\beta$ and IL-6 and IL-6, have been shown to promote EOC genesis, growth and progression (Nowak et al., 2010a, Clendenen et al., 2011).

The most important hypothesis to arise about EOC carcinogenesis was the ovulation theory, which relates ovarian cancer risk to incessant ovulation. To support this hypothesis, there is growing interest in the etiologic role of inflammation that accompanies each ovulation 
(Landen et al., 2008). Ovarian surface epithelium adjacent to the site of ovulation may be exposed to inflammatory and oxidative status with consequent risk of malignant transformation. Intriguingly, the same ovulatory process together with the repair steps immediately after liberation of the ovum, are characterized by the generation of an enormous amount of cytokines/chemokines and matrix-remodeling enzymes, including prostaglandins, bioactive eicosanoids, plasminogen activators, collagenases, interleukins (ILs), TNF-a and various growth factors (Macciò et al., 1994) as well as by the recruitment of activated immune cells to the wounded epithelial surface, entailing the global activation of the pro-inflammatory network. Recently, it has been hypothesized that high grade serous ovarian cancer, endometrioid and clear cell cancers arise from fallopian tube epithelium and share a common pathogenic mechanism, i.e. iron-induced oxidative stress derived from retrograde menstruation. Fimbriae floating in bloody peritoneal fluid are exposed to the action of catalytic iron and to the genotoxic effect of reactive oxygen species, generated from haemolysis of erythrocytes by pelvic activated macrophages and by the cytokines secreted from themselves. In summary, both incessant ovulation and oxido-reductive fallopian tube epithelial damage hypotheses have provided evidence that inflammatory responses induced under physiological conditions may foster the development of EOC.

A growing body of evidence suggests that, although genetic events in the tumour cells themselves are definitely crucial, host and stromal factors in the tumour microenvironment are equally important. A clinically overt tumour includes not only cancer cells but also matrix components, stromal cells and inflammatory cells. In particular, in EOC peritoneal and stromal alterations alongside with their lymphomocytes components and associated cytokines may be permissive for cancer growth and spread. Likewise, cytokine production also by tumour cells themselves can both promote their growth and inhibits apoptosis in an autocrine manner. Therefore, inflammation seems to contribute to every step of carcinogenesis, including tumour initiation, promotion, and progression. On the other hand, tumour cells can produce immunogenic proteins that are recognized as foreign, potentially thus inducing an antineoplastic immune response. Actually, the tumour uses these immunological interactions to evade recognition and destruction by immune cells, i.e. Fas ligand production to induce lymphocyte apoptosis (Mantovani et al., 1999a) and HLA-G secretion to inhibit natural-killer cell activity. Then, although the importance of the host antitumor immune response, as demonstrated by the finding that increased T-cell infiltration into the tumour is associated with improved survival (Zhang et al., 2003), the real role of immune system in containing tumour growth remains to be fully defined (Landen, 2008).

\section{Proinflammatory cytokines in the progression of EOC}

Components of the inflammatory pathway, including free radicals, cytokines, NF- $\mathrm{kB}$, signal transducer and activator of transcription-3 (STAT-3), inducible nitric oxide synthase (iNOS), cyclooxygenase-2 (COX-2), prostaglandins, and vascular endothelial growth factor (VEGF) have been shown to contribute to the development of various malignancies, including EOC. In particular, COX-2 was found to be highly expressed in non-mucinous ovarian cancers, and its expression was correlated with poor prognostic factors, such as stage, residual disease status and presence of ascites (Ferrandina et al. 2002a). Consistently with this 
hypothesis, patients with chronic aspirin, nonsteroidal anti-inflammatory drug, or acetaminophen use have a reduced risk of EOC (Altinoz \& Korkmaz, 2004).

\subsection{Cytokines as cancer growth factors}

Multiple genetic alterations are implicated in ovarian carcinogenesis, but clinical and genetic evidence support two wide categories of EOC carcinogenesis: those of low-grade and highgrade pathways. Gene and protein analyses of tumours of these two different subtypes also suggest different pathogenesis: K-Ras, BRAF, and PTEN mutations are more frequently observed in low-grade tumours, whereas P53 mutation is predominantly present in highgrade tumours, but rarely in other subtypes or low malignant potential (LMP) tumors. Moreover, HER2 and AKT are overexpressed in high-grade carcinomas but rarely in lowgrade and LMP tumours. Overexpression of human leukocyte antigen-G (HLA-G), which may provide a mechanism of immune escape for the tumour, has been noted in a high percentage of high-grade carcinomas but is absent in low-grade or LMP neoplasma (Landen, 2008). Moreover, the new proposed histological classification of EOC in type I slow growing tumours and type II rapidly growing and highly aggressive tumours is accompanied by a specific expression of the inflammatory markers: glucose transporter protein-1 (Glut-1), inducible nitric oxide synthase (iNOS), COX-1, COX-2) and nuclear factor kappa B. In detail, overexpression of COX-1, COX-2, iNOS, and Glut-1 was significantly higher in type II tumours and was associated with a poorer median survival as compared with those with type I tumours. Therefore, the distinct expression of these markers may explain the different biologic behaviour of these 2 tumour types and provide targets for therapy (Ali-Fehmi et al., 2011).

Although EOC can be subdivided by grade, their histological subtypes also differ. Serous, endometrioid, and mucinous adenocarcinomas have difference in clinical outcomes even if not as dramatic as those between high- and low-grade cancers. However, genomic studies have demonstrated that mucinous adenocarcinomas often harbour mutations and have peculiar gene expression similar to LMP tumours and to benign cystadenomas. Specifically, mutations in K-RAS have been described in borderline, low-grade tumours and mucinous adenocarcinomas, but are very rare in high-grade serous carcinomas. Moreover, endometrioid adenocarcinomas harbour PTEN mutations (similar to endometrioid tumours of the uterine endometrium) more frequently than do serous or mucinous subtype. The discovery of these genetic mutations allowed hypothesizing a model of multistep carcinogenesis of ovarian cancer (Landen, 2008). To become a clinically evident tumour ovarian cancer cells must overcome many protective mechanisms: these include unchecked proliferation, evading apoptosis, angiogenesis, stromal invasion, separation and survival away from the primary tumour, and implantation and growth within new tissues. Within the dual pathway model, it is clear that the tumour cell and its environment must acquire the above characteristics. Although the order in which these occur is likely variable, early alterations in dominant genes may dictate the specific path that is followed, such as K-RAS leading to an LMP tumour and early occurrence of a p53 alteration leading to genetic instability and rapid progression to a high-grade phenotype. Many researchers show a role for inflammation in tumour initiation, promotion, progression and metastatisation. In particular most studies focused their attention to IL-6 signalling which seems to play the main role (Lane et al., 2011). IL-6 is one of the major immunoregulatory cytokines present in the EOC microenvironment. Both ovarian cancer cells and tumor-associated macrophages 
produce IL-6, and it is to date known that high serum levels of IL-6 are related with specific immune and metabolic alterations which finally lead to cancer cachexia, the main cause of death of EOC patients. IL-6 has also been demonstrated to be involved in autocrine growth of ovarian cancer cells [19-21] as well as in tumorigenesis and progression of ovarian cancer cells particularly by increasing their capacity to secrete matrix metalloproteinase (MMP)-9 (Rabinovich et al., 2007). Then, IL-6 could stimulate the proliferation of tumour cells either directly and/or by promoting angiogenesis. In fact, IL-6 has an important role, precisely through tumour angiogenesis, in promoting the development of ascites as well as the spread of ovarian cancer thus leading to fast progression and short survival. (Lane, 2011; Lo, 2011). The high levels of IL-6 enhance the immune suppressive status of the tumour microenvironment by inhibiting IL-2 synthesis, T cell activation and proliferation, and promoting lymphocytes apoptosis (Macciò, 1998; Mantovani, 1999a). Furthermore, IL-6 may divert the immune response from Th1 towards a suppressive Th2 response although controversial data have been reported. Another inflammatory cytokine TNF- $\alpha$ that is constitutively expressed in the malignant ovarian surface epithelium generates and sustains a network of other mediators that promote tumour growth and peritoneal spread. Constitutive production of TNF-a is associated with greater release of IL-6 itself as well as other chemokines as: CCL2 and CXCL12, macrophage migration-inhibitory factor (MIF) and VEGF. In turn, these factors may act in an autocrine/paracrine manner to promote colonization of the peritoneum and neovascularization of developing tumour deposits. Moreover, also estrogens by the modulation of proinflammatory cytokines, and in particular IL-6, are involved in regulating the growth and progression of EOC. Estrogens not only enhance cytokines production but also modulate the expression of their receptors. In turn, IL-6 and IL-8 also promote ovarian cancer cells growth through an oestrogen receptor pathway. Therefore, these findings provide a novel mechanism that oestrogens, IL-6 and IL-8 may form a common amplifying signalling cascade to modulate ovarian cancer cells growth and progression (Yang et al., 2009).

From what has been written it can be deduced that IL-6 is the cytokine mainly involved in EOC carcinogenesis and progression. IL-6 is a $26-\mathrm{kDa}$ glycopeptide whose gene is found on chromosome 7, produced by antigen presenting cells (APCs) such as dendritic cells, macrophages and B cells among other cells of the haematopoietic system. It is also produced by a variety of non-haematopoietic cells including keratinocytes, fibroblasts, epithelial cells, and neoplastic cells. IL-6 gene transcription is induced in many different normal tissues in response to stimuli, such as RNA and DNA virus infection, bacterial endotoxin, lipopolysaccharide and other inflammatory cytokines as TNF- $\alpha$, IL-1, and platelet-derived growth factor (PDGF) and the interferons (IFNs). It has been previously named hepatocytestimulating factor, cytotoxic T-cell differentiation factor, B-cell differentiation factor, B-cell stimulatory factor 2, hybridoma/plasmacytoma growth factor, monocyte granulocyte inducer type 2 and thrombopoietin. The many names reflect the pleiotropism of IL-6. IL-6 affects virtually every organ, most notably the immune system and in particular, it is an essential factor for the normal development and function of both $\mathrm{T}$ and $\mathrm{B}$ lymphocytes and has broad actions on cells of the haematopoietic system. Efficient induction of the IL-6 promoter requires the interaction of several transcription factors, including the CAAT enhancer-binding protein (C/EBP) family members and nuclear factor $k B(N F-k B)$. Nuclear factor for IL-6 (NF-IL6, C/EBP-b) and NF-kB interact with each other to synergistically upregulate the IL-6 promoter, just like NF-IL6 (C/EBP-b) and NF-IL6b (C/EBP-d). The IL-6 
promoter is inhibited by $\mathrm{p} 53$ and the retinoblastoma $(\mathrm{Rb})$ gene product. The overexpression of IL-6 in many malignancies may occur as a result of the loss of one of these negative regulators of transcription.

The physiological activity of IL-6 is complex, including both pro-inflammatory and antiinflammatory effects in the immune system. In fact, relative to its specific immunomodulating capacity, IL-6 is an activator or an inhibitor of T-cell responses, depending to the length of time of its activities. This combination of pro-inflammatory and anti-inflammatory effects suggests that IL-6 may play a role in regulating the control of immune system activation during the different phases of EOC evolution. IL- 6 modulates the transcription of several liver-specific genes during acute inflammatory states, particularly Creactive protein (CRP) and hepcidin. IL-6 can also up-regulates the multidrug resistance 1 (MDR-1) gene through activation of NF-IL6, which, in turn, transactivates the MDR-1 gene through a Y-box motif. IL-6 blood levels are high in numerous infectious, inflammatory, and autoimmune diseases and in cancer in association with increased synthesis of other cytokines and specific immunological challenge. Human diseases that involve prolonged inflammation and in particularly advanced EOC frequently exhibit cachexia with loss of muscle mass and IL-6 seems to be the key mediator of these processes as well. It is noteworthy that high circulating levels of IL-6 have also been linked to insulin resistance, high body mass index and obesity. IL-6 also exerts its effects on the central nervous system, where it regulates glial cell activation and modulate mood as well as induce severe depressive symptoms.

IL-6 signals primarily by its binding to a specific receptor (IL-6R) which is a member of the Class I cytokine receptor family. Functional Class I receptors contain high-affinity ligandbinding components and signal-transducing components, and are thus multichain receptor complexes that often share the signal-transducing element. Then, IL-6 signals through a protein complex including the membrane-bound non-signalling a-receptor subunit (IL-6R achain gp80 or CD126) and two signal-transducing gp130 subunits (IL6-R $\beta$-chain gp130 or CD130), this second chain of the receptor resulting in the formation of high-avidity IL-6 binding receptors (Lo, 2011). More precisely: the ligand-binding portion of the IL-6R is an 80-kDa molecule associates directly with IL-6 that exists both in a membrane-bound and a soluble form; the signal transducing component of the IL-6R complex is glycoprotein 130 (gp130), sometimes called IL-6Rb-chain. The gp130 functions as an affinity converter because the resulting affinity of IL-6 for the ternary complex is approximately $10^{-11} \mathrm{M}$ instead of $10^{-9} \mathrm{M}$ for IL-6R. While gp130 is expressed ubiquitously, gp80 is physiologically mainly expressed on hepatocytes and specialized subsets of leukocytes, including neutrophils, monocytes/macrophages, and T and B lymphocytes. However, IL-6 can also signal via a soluble receptor (sIL-6R or gp55 chain) that lacks the transmembrane and cytoplasmatic components. Soluble IL-6R (sIL-6R) can be generated by two mechanisms: 1) Metalloproteinase mediated cleavage ("shedding") of the membrane bound form of the IL$6 R$ and 2) expression of an alternatively spliced IL-6R variant that lacks the transmembrane domain. Neutrophils and macrophages in addition to some cell lines have been shown to produce sIL-6R. Activated sIL-6R binds to membrane-bound gp130 subunits in a process known as trans-signalling. Therefore, unlike other soluble cytokine receptors, which are generally antagonists, sIL-6R is an agonist molecule, promoting IL-6 activity. This ability may explain a possible activation of gp130 despite the lack of gp80, if sIL-6R molecules 
circulate in great quantity, as demonstrated in certain pathological states. Accordingly, it was observed that cells lacking IL-6R expression are responsive to IL-6 stimulation especially during inflammatory conditions. As demonstrated in EOC, this alternate pathway serves as the major signalling in inducing endothelial hyperpermeability and increasing transendothelial migration of cancer cells, thus contributing to cancer progression. Moreover, elevated levels of sIL-6R in malignant ascites from ovarian cancer patients are associated with poor prognosis (Lo, Cancer Res 2011; 7: 424-34). The increase of IL6R expression as well as of the soluble spliced variant of IL6R in malignant ovarian tumours are regulated by cancerassociated inflammation (Rath et al., 2010). Therefore, in advanced EOC IL6R is overexpressed mainly because of increases in a sIL6R variant, which can influence its evolution and prognosis. In addition to sIL-6R, soluble gp130 (sgp130) also exists in human serum and acts as an antagonist of the IL-6/ sIL-6R complex.

Once IL-6 binds its receptor and gp130 homodimerization occurs, a signalling cascade is triggered. X-Ray crystallography has shown that two heterotrimers of IL-6, IL-6R and gp130 associate to form a hexameric complex. Through formation of this complex, members of the cytoplasmic Janus kinase (Jak) family of tyrosine kinases bind to gp130 inducing phosphorylation of downstream targets. The Janus kinases activation is followed by the recruitment of signal transducers and activators of transcriptions (STATs). One phosphorylated, STATs translocate to the nucleus where they promote gene transcription. IL-6R stimulation also recruits other signal transduction molecules, including SH2 domaincontaining tyrosine phosphatase (SHP2) and suppressor of cytokine signalling (SOCS). Both SHP2 and SOCS may subsequently down-regulate IL-6 signalling. Jak1 is thought to be the most relevant for IL-6 signalling although Jak2 and Tyk2 also transduce some of the IL-6 signals. In some instances, IL-6 acts with other factors, such as heparin-binding epithelial growth factor and hepatocyte growth factor in controlling proliferation and function of various cell types. Blocking IL-6 by specific anti-receptor drugs may thus be of benefit in many pathological situations.

The best-described substrate for Jaks in IL-6 signaling is the STAT 3, a transcription factor that in its inactive form remains in the cytoplasm but after phosphorylation forms homodimers that are actively being transported to the nucleus to induce gene transcription. Increasing evidence indicates that tumour cells express constitutively activated Stat proteins, particularly STAT3, independent of dysregulation of upstream molecules, disabled inhibitory mechanisms or identifiable ligand stimulation. Stat3 overexpression also may promote cell proliferation and transformation into a tumour phenotype. Overexpression and overactivation of Stat3 is found in EOC tissue and the constitutive activation of Stat3 signalling pathway may play an important role in the invasion and prognosis. The expressions of Stat3 and phosphorylated (p)-Stat3 in EOC are significantly higher than in normal ovarian epithelial tissues or benign ovarian tumour and the expression of Stat3 protein is highly correlated with the expression of p-Stat3 protein. The nuclear localization of p-Stat3 predicts a poor prognosis: in fact, pSTAT3 expression is significantly correlated with disease stage, degree of differentiation and lymph node metastasis (Min \& Wei-hong, 2009). Recent studies suggest that STAT3 is a key factor for EOC chemoresistance, showing that STAT3 decoy oligodeoxynucleotides (ODN), its specific antagonist, inhibited cancer cell invasive power and enhanced sensitivity to paclitaxel. The mechanism involves the inhibition of EMMPRIN, P-gp, and pAkt by STAT3 decoy ODN. These three proteins are 
probably the target proteins of STAT3 (Zhang, 2010). Increased levels of pSTAT3 are correlated with increased expression of HER-2/neu, EGFR and proliferation but not apoptosis markers. Unlike other molecules involved in oncogenesis, no genetic mutations or amplifications have been identified for STAT3, suggesting that persistent STAT3 activity is caused mostly by the dysregulation of upstream molecules, such as receptors with intrinsic tyrosine kinase activity (e.g., EGFR or HER-2/neu) and, in particular, endogenous or exogenous IL-6. Moreover, the regulation and functions of Stat proteins are highly dependent on the cell type, the activating stimulus and the cellular context, especially the activity of other signalling pathways and transcription factors that interact with the Stat proteins. Consequently, depending on the cellular context, STAT3 may mediate conflicting responses in terms of cell proliferation, differentiation or apoptosis. For example, the concurrent coexpression of dominant-negative STAT3 and the oncoprotein Ras does not arrest Ras-induced transformation, suggesting that STAT3 signalling is only one of several pathways required for cell transformation induced by this oncogenic tyrosine kinase. In addition, STAT3 demonstrates a histotype-specific pattern of expression. High levels of expression were observed more commonly in those histotypes with aggressive biologic behaviour (undifferentiated, clear cell, and serous carcinomas) than in those histotypes with less aggressive behaviour (mucinous and endometrioid carcinomas).

Results from a recent study (Saydmohammed et al., 2010) confirm that IL-6 secretion increases during malignant progression of ovarian epithelial cells and found that IL-6 expression levels are not always correlated with the expression or subcellular location of pSTAT3 in ovarian carcinoma, supporting the finding that IL-6 is involved in other signalling pathways, independent of STAT3. Moreover, given the observations that cancer cells can constitutively express STAT3 in the absence of stimulation by any known ligand and that expression of STAT3 is higher in ovarian carcinoma than in normal ovarian tissue, it is possible to speculate that the constitutive activation of STAT3 in ovarian cancer cells could be because of aberrant EGFR signalling. In agreement with this possibility, it has been observed a significant correlation between high levels of pSTAT3 expression and the overexpression of EGFR and HER-2/neu in EOC (Bast et al., 1993). Alternatively, the constitutive activation of STAT3 in EOC may be caused by the elevation of Src and focal adhesion kinase levels (Rosen et al., 2006) More recently, a significant activation of both STAT-3 and its upstream activator JAK-2, has been demonstrate in high-grade ovarian carcinomas compared with normal ovaries and benign tumours. The association between STAT3 activation and migratory phenotype of ovarian cancer cells was investigated by EGFinduced epithelial-mesenchymal transition (EMT) in ovarian cancer cell lines. Ligand activation of EGFR induced a fibroblast-like morphology and migratory phenotype, consistent with the upregulation of mesenchyme-associated $\mathrm{N}$-cadherin, vimentin and nuclear translocation of beta-catenin. This occurred concomitantly with activation of the downstream JAK2/STAT3 pathway. The cell lines expressed the IL-6R and treatment with EGF resulted in enhanced IL-6 expression and release in the serum-free medium. Exogenous addition of IL-6 stimulated STAT3 activation and enhanced migration. Blocking antibodies against IL-6R inhibited both IL-6 production and EGF- and IL-6-induced migration. Specific inhibition of STAT3 activation by a JAK2-specific inhibitor blocked STAT3 phosphorylation, cell motility, induction of N-cadherin and vimentin expression and IL6 production. These data suggest that the activated status of STAT3 in high-grade EOC may occur directly through activation of 
EGFR or IL-6R or indirectly through induction of IL-6R signalling. Such activation of STAT3 suggests a rationale for a combination of anti-STAT3 and EGFR/IL-6R therapy to suppress the peritoneal spread of ovarian cancer (Colomiere et al., 2009).

In addition to STAT3 also the Ras protein can be activated in response to IL-6. After Ras activation, hyperphosphorylation of mitogen-activated protein kinase (MAPK) occurs as well as an increase in its serine/threonine kinase activity. MAPK then phosphorylates the NF-IL6 transcription factor on serine 231 and threonine 235, a process that is essential for DNA binding. NF-IL6 has a basic leucine zipper motif and is a member of the C/EBP family of transcription factors. NF-IL6 activates the promoter regions of various acute-phase protein genes in the liver. Thus, when IL-6 binds to a cell through IL-6Ra/gp130 complexes, a series of events takes place that leads to the activation of STATs and NF-IL6, switching on target genes. OSE cells immortalized with mutant H-Ras or K-Ras lead to cells that grow slowly but progressively with serous papillary histology in the peritoneal cavity. Gene expression profile analysis of these transformed cells showed an increased expression of several cytokines, mainly IL-6, which are up regulated by the NF-kB pathway. Each of these cytokines might provide targets for therapeutic intervention in EOC with RAS mutation.

\subsection{Cytokines and modulation of immune system}

The host immune response comprises a multitude of highly developed interconnected biological processes involving both cellular and humoral responses that cooperate to eliminate foreign bodies and repair the site of injury. The innate arm of the immune activity provides rapid reactions prior to the development of highly specific adaptive responses. In the context of a malignant tumour, many of the suppressive and stimulatory properties of innate immunity may influence tumour progression in both positive and negative ways. The activation of the cell-mediated immunity by macrophages, T lymphocytes, and natural killer cells has been suggested as a specific mechanism performed by the body to counteract oncogenesis and tumour growth. During their activation processes these cells release several soluble factors (cytokines) that send stimulatory or inhibitory signals to the different immune cell types. Interleukin-1, IL-2 and TNF- $\alpha$ are the main mediators of cell-mediated immune response. Interleukin-1 and TNF-a are potent inductors of IL-6 that, in turn, regulates their production, acts as a second signal for the production of IL-2 and induces on cytotoxic T lymphocytes the expression of IL-2 receptor (RIL-2). IL-2 is the key cytokine in the regulation of the antineoplastic immunity. The activity of IL-2 is strictly dependent on its binding to specific membrane receptor (IL-2R). Lymphocyte activation is followed by an increased expression of IL-2R and release of its a subunit from the membrane receptor in a soluble form (sIL-2R). Hence, sIL-2R serum levels provide direct evidence of immune system activation. Then, the synergistic effect of IL-2 and other cytokines deriving from the activated immune system may play an active role in the cytotoxic attack against tumour by counteracting neoplastic cells growth. However, some cytokines, such as IL-1, IL-6 and TNF-a may favour tumour progression. Indeed, several studies of our research group have shown in vitro that the immune system of EOC patients is inefficient to various mitogen stimuli in terms of lymphocyte proliferative response and that the severity of the immune deficit is proportionate to the stage of disease and to the performance status (PS) of patients (Mantovani et al., 2000, 2003). The reduced lymphocytes proliferative response to mitogens, such as phytohaemagglutinin (PHA) and anti-CD3 monoclonal antibody (mAb), must be considered as an index of more complex functional alterations. In fact, these mitogens 
induce in vitro a number of phenomena similar to those that follow antigenic activity in vivo. The secretion of macrophagic cytokines, the production of IL-2 by CD4+ lymphocytes and the RIL-2 expression on lymphocyte membrane are the defining moments of these events. For these reasons, the entity of the lymphocyte blastic response depends on the quantity of cytokines produced, the number of RIL-2 expressed and the physiologic interaction of IL-2 with its receptor. Lymphocytes inability both to produce adequate quantities of IL-2 and to express physiological amount of RIL-2 seems to be the crucial feature of this specific lymphocyte functional deficit in EOC patients. In our studies, patients peripheral blood mononuclear cells (PBMC) proliferative response to $\mathrm{PHA}$, anti-CD3 mAb and human recombinant IL-2 (HurIL-2) alone was significantly lower in comparison to controls and it was not modified by the addition of human recombinant IL-2 (HurIL-2) to the culture media. Furthermore, also the expression of CD25 and CD122 subunits of membrane-bound IL-2R on patients' PBMC after stimulation with PHA or CD3mAb was lower than that seen in controls (Macciò et al., 1998). A very important finding of our researches highlights that this impairment of $\mathrm{T}$ cells response was associated with increased circulating levels of proinflammatory cytokines (IL-1a, IL-1 $\beta$, IL-6, TNF- $\alpha$ ) and other mediators of inflammation such as fibrinogen, CRP and sIL-2R (Figure 1).

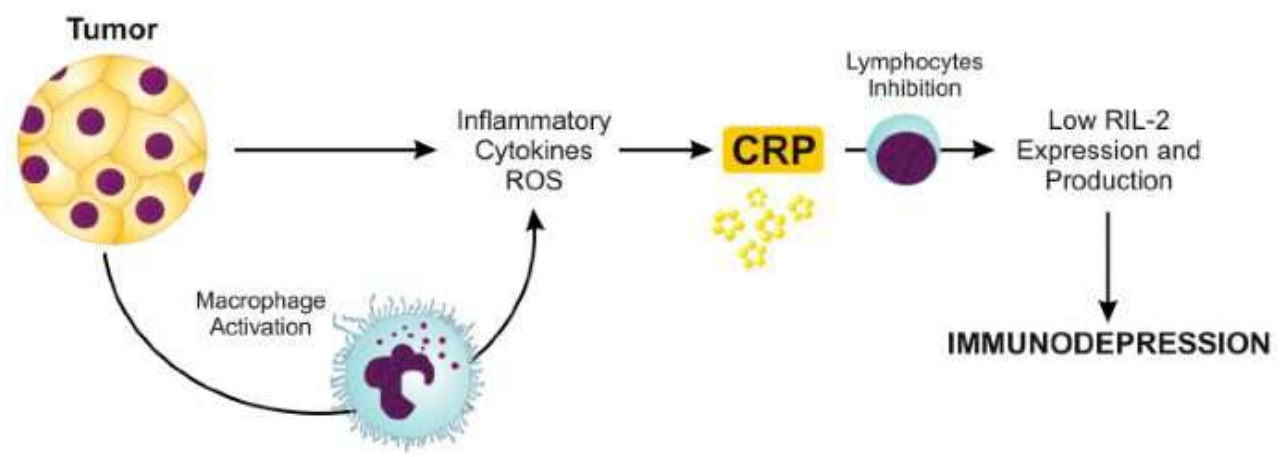

Fig. 1. Aspecific activation of immune system during the evolution of the ovarian cancer leads to immunodepression associated to high serum levels of inflammatory cytokines and acute phase proteins. Abbreviations: ROS, Reactive Oxygen Species, RIL-2, IL-2 receptor, CRP, C-reactive protein.

In particular, it is extremely interesting that IL-6 and CRP have been shown to be able to suppress $\mathrm{T}$ cell responses and several studies suggested that they might interfere with the immunological mechanisms underlying the antitumor activity of IL-2. Moreover, it is known that CRP, typically induced by IL-6, is involved in the binding of complement to cytotoxic CD3+ cells and plays a key role in the inhibition of cytotoxic activity of NK cells. Then, IL-6 can be an activator or an inhibitor of T-cell responses, depending its effects by the time and duration of its activity. This interaction of pro-inflammatory and antiinflammatory activities suggests that IL-6 may play a role in regulating the control of immune system activation during the different phases of in EOC progression. A widely accepted model of tumour and immune cell interaction, termed immunoediting, describes an initial restriction of tumour cell growth, but maintains that the immune system ultimately selects for tumour cells with reduced immunogenicity that subsequently prevail 
over the host immune system. Therefore, whereas the immune system may initially be protective against tumour development, its efficacy may diminish over time and it may ultimately facilitate tumour progression. Indeed, the mechanisms by which the tumour can evade immune system control are manifold. Despite immune-cells have for long been known for their roles primarily in immune cancer surveillance, many tumour cell types secrete immunosuppressive cytokines such as transforming growth factor-beta, IL-6, IL-10 and IL-13, and chemokines that can also recruit cells that negatively regulate immunity such as T-regulatory cells, myeloid suppressor cells, NK cells and macrophage subsets (RobinsonSmith et al., 2007). Jeannin P et al. in a very recent work (Jeannin et al., 2011) reported that ovarian cancer ascites switched monocyte differentiation into tumour-associated macrophages (TAM)-like cells, that exhibit most phenotypic and functional characteristics of TAMs, suggesting that soluble mediators are involved in the differentiation of monocytes into TAM-like cells. TAMs, the most abundant immunosuppressive myeloid cells in the tumour microenvironment, exhibit an IL-10 (high) and IL-12 (low) profile called M2, opposite to the immunostimulatory M1. The same authors observed that the leukaemiainhibitory factor and IL-6, present at high concentrations in ovarian cancer ascites, skew monocyte differentiation into TAM-like cells by increasing macrophage colony-stimulating factor consumption. These data reveal a new tumour-escape mechanism associated with TAMs generation through an IL-6 mediated effect. An interesting published study by Nowak et al. confirmed that in the presence of autologous ovarian cancer cells, peripheral blood mononuclear cells from patients with advanced EOC produced higher amount of immunosuppressive (Il-10, TGF-beta) and proinflammatory (IL-6) cytokines with downregulation of T cells response (Nowak et al., 2010b). In the context of EOC, two specific leukocyte subsets have been demonstrated to significantly promote tumour growth: regulatory $\mathrm{T}$ cells (Tregs) and pro-angiogenic/immunosuppressive myeloid cells, the latter exhibiting the phenotypic attributed of macrophages (Cubillos-Ruiz et al., 2010). Globally, all ovarian cancer-associated myeloid cell subsets impair the function of anti-tumour T cells, (Scarlett et al., 2009) the only element in the ovarian cancer microenvironment known to exert clinically relevant spontaneous immune pressure against tumour progression. The accumulation of tumour Tregs predicts poor survival in EOC patients. Curiel and colleagues (Curiel et al., 2004) first demonstrated a crucial role for Tregs in ovarian cancer-mediated immunosuppression. They showed that solid tumour masses and malignant ascites of human ovarian cancer accumulate variable levels of Tregs (CD3+CD4+CD25+ GITR+CTLA4+CCR7+FoxP3hi), while non-malignant ascites or normal ovaries did not contain a significant proportion of these cells. Interestingly, Tregs were found to be specifically recruited to tumour locations via CCL22, a cytokine expressed by tumour cells and microenvironmental myeloid cells. Ovarian tumours and tumour microenvironment macrophages are major sources of CCL22. Tregs isolated from ovarian cancer ascites were functionally active, as they inhibited the proliferation of autologous $\mathrm{T}$ cells stimulated in vitro with DCs pulsed with tumour antigens, and also prevented the anti-tumour activity of adoptively transferred $\mathrm{T}$ cells. Giuntoli et al. reported that a high CD4+/CD8+ ratio in ascites, which may indicate the presence of Tregs, is associated with poor outcome (Giuntoli et al., 2009). Other studies investigating the significance of the role of intratumoral infiltrates (TIL) or tumour associated lymphocytes (TAL) in these events have been reported. By contrast, there is accumulating evidence that the presence both of TIL or TAL, such as those found in neoplastic effusions, is quantitatively related with improved clinical outcome in ovarian cancer (Kim et al., 2009). In fact, recent studies report 
on the infiltration of ovarian cancer by both CD4+ and CD8+ TILs and show a positive correlation between T-cell infiltration and prognosis (Yigit et al. 2010). Napoletano et al. demonstrated that primary debulking in ovarian cancer is associated with a reduction of circulating Tregs and an increase in CD8+ T-cell function (Napoletano et al., 2010). Leffers at al. reported that a high TIL/Treg ratio independently predicts increased survival and suggest that it is not so much the presence of Treg as the presence of TIL in general to be responsible for the observed survival effect (Leffers et al. 2009).

A central mechanism whereby both TIL and/or TAL contribute to invasive proliferation of tumour cells is through the production of the cytokines and chemokines that increase both the migration and survival of tumour cells. These cytokines present in the blood and in large quantities in neoplastic effusions can also be produced by cancer cells and have been associated with prognosis in EOC (Gavalas et al., 2010).

In conclusion the development of EOC is associated with changes in the peritoneal cavity microenvironment. Immune cells in the ovarian stromal microenvironment play an important role in ovarian tumorigenesis and progression (Wertel et al., 2011). In turn, tumour cells develop several mechanisms to evade anti-tumour immunity by developing an immunosuppressive microenvironment by the production of different factors (cytokines), which impairs differentiation, maturation, and function of antigen-presenting cells. Once transformed ovarian epithelial cells develop an immunoediting process occurs in which immune cells and their mediators dictate the growth and progression of EOC (Thompson \& Mok, 2009). Then, as described above chronic inflammation is associated with initiation and/or progression of the most common EOC types and the balance between pro- and anti-inflammatory cytokines is critical for host immune response to tumours.

\section{Proinflammatory cytokines and prognosis}

Several studies, including some from our group (Macciò et al., 1998, 2009), demonstrated the correlation existing between the severity of chronic inflammation, advanced stage and poor outcome in patients with epithelial ovarian cancer. Epithelial ovarian cancer is an immunogenic tumour and exploits many suppressive ways to escape immune eradication. High circulating levels of proinflammatory cytokines, such as IL-1, Il-6, and TNF- $\alpha$ have been found in EOC patients with advanced stage of disease and an unfavourable prognosis. The prognostic role of various cytokines has been studied, but no absolutely firm conclusions can be drawn so far. It is likely that cytokines involved in Th1 response predict for better prognosis, while the opposite is expected in those associated with Th2 response. Moreover, proinflammatory cytokines play an important role in the mechanisms inducing the complex clinical condition known as cancer-related anorexia/cachexia (CACS). One of the metabolic changes present in this syndrome is the hepatic synthesis of C-reactive protein (CRP). High serum levels of CRP are associated with a poor prognosis in EOC patients and can negatively influence the therapeutic response to HurIL-2. This is extremely important since IL-2 initiates the activation of T and NK cells and it is also essential for the maintenance of self-tolerance through generation and maintenance of Tregs or by activation-induced cell death to eliminate self reactive T cells. Interestingly, IL-6 is a potent inducer of CRP exerting its regulatory effect 
on CRP synthesis at the pretranslational level. IL-6 levels have been shown to be increased in advanced ovarian cancer patients' serum and to correlate with poor prognosis and reduced overall survival (Scambia et al., 1995). Elevated levels are also present in malignant ascites from EOC patients (Plante et al., 1994) and a positive correlation has been found between IL- 6 concentration in ascites and residual disease after debulking. Additionally, IL-6 levels are remarkably higher at recurrence compared to primary advanced disease, thus opening an opportunity for inhibition of IL-6 expression in the prevention of recurrence. EOC is known to spread primarily by tumour cell implantations in peritoneal cavity. Therefore, ascites may be an ideal fluid compartment to unravel the immune status of the peritoneal cavity (Mantovani et al, 1999, 1997). Recently, Yigit R et al. (Yigit et al., 2011) observed high expression of pro-inflammatory cytokines IL-6, IL-8 and immune suppressive cytokines IL-10, CCL22 and TGF- $\beta$ in most samples of ovarian cancer ascites whereas Th1 (IL-12p70, IFN- $\gamma$ ) and Th2 (IL-4, IL-5) cytokines were only detectable in few samples. TGF- $\beta$ was only detected in latent form, questioning its immune suppressive role. At advanced stage, they also observed a negative correlation with CCL22 levels and Th1/2 cytokine expression. A cytokine that seems to be heavily involved in tumour immunosuppression is the transforming growth factor beta (TGF- $\beta$ ), a protein that affects proliferation, activation, and differentiation of immune cells and inhibits antitumor immune response. In cancer cells, the production of TGF- $\beta$ is increased and, in turn, raises their proteolytic activity and binding to cell adhesion molecules in the extracellular matrix. TGF- $\beta$ can also convert effector $\mathrm{T}$ cells into Tregs. It has been reported that it can also promote angiogenesis and that this process can be blocked by anti-TGF- $\beta$ antibodies. TGF- $\beta$ blockade almost completely eradicate ascites formation and significantly inhibit the expression of VEGF, which is the major contributor to ascites formation. At the same time, TGF- $\beta$ blockade prevent 'abnormalization' of diaphragm lymphatic vessels and improve ascites drainage (Liao et al., 2011). Also TNF- $\alpha$ is produced by tumour cells and can induce autocrine proliferation and disease progression in ovarian cancer. The autocrine action of TNFa may have direct effects on tumour cell spread via acting on the chemokine receptor CXCR4 and stimulating new blood vessel formation in the peritoneum by inducing expression of VEGF and CXCL12. In contrast, TNF-a levels have also been inversely correlated with the presence of CD4+ CD25+ cells, and have been shown to directly downregulate Tregs. This might indicate a favourable effect of this cytokine on prognosis and underlines the complexity of the functions that each of these factors may possess. Then, reports on whether TNF- $\alpha$ is a signature of poor or better prognosis vary. Another cytokine that was shown to be associated with the growth of cancer cells and tumour proliferation is IL-1. A family of proteins called chemokines (CC) may also be influencing cellular composition in biological fluids. Recent studies have demonstrated the presence of mRNA for CCL2, CCL3, CCL4, and CCL5 in EOC by in situ hybridization. Moreover, CCL5 has been shown to be secreted by CD4+ T cells, recruits CCR5+ dendritic cells to the tumour location, and activates them through CD40-CD40L interactions. The newly matured dendritic cells prime tumour-specific CD8+ cells thus providing with long-term protection. Also in the protein-rich ascitic fluid, different chemokine molecules are expressed, with CCL2 being the predominant one. In addition, chemokine stromal-derived factor-1 (CXCL-1) induced the migration of plasmacytoid dendritic cells (PDC) into the tumour microenvironment in cases of ovarian 
cancer and induced delivery of survival signals to PDC. In turn, the tumour microenvironmental PDC induced IL-10 expressing Tregs, which are correlated to poor prognosis and shorter progression-free survival. In the case of Tregs it has been exhibited that CCL22 plays a central role in inducing influx of these cells into tumour sites by binding to CCR4 that is expressed on Treg surface. Interferon gamma (IFN- $\gamma$ ) plays a stimulatory role for macrophages turning them from immunosuppressive to immunostimulatory cells. It also skewed monocyte differentiation from associatedassociated macrophages (TAM) like cells to M1-polarized immunostimulatory macrophages. Taken together these data show that IFN- $\gamma$ overcomes TAM-induced immunosuppression by preventing TAM generation and functions. Furthermore, cytokines such as IL-18 and stroma derived factor 1 (SDF-1) have been shown to be correlated with poor prognosis in ovarian cancer patients, but further studies are required to fully evaluate them in the tumour microenvironment and the periphery.

\subsection{Inflammation and metabolic changes}

In the course of its evolution cancer induces in the host changes of the immune system and energy metabolism that affect its clinical conditions so deeply that in some cases they are responsible for patient's death. Several symptoms are associated to these events and involve various organs and systems:

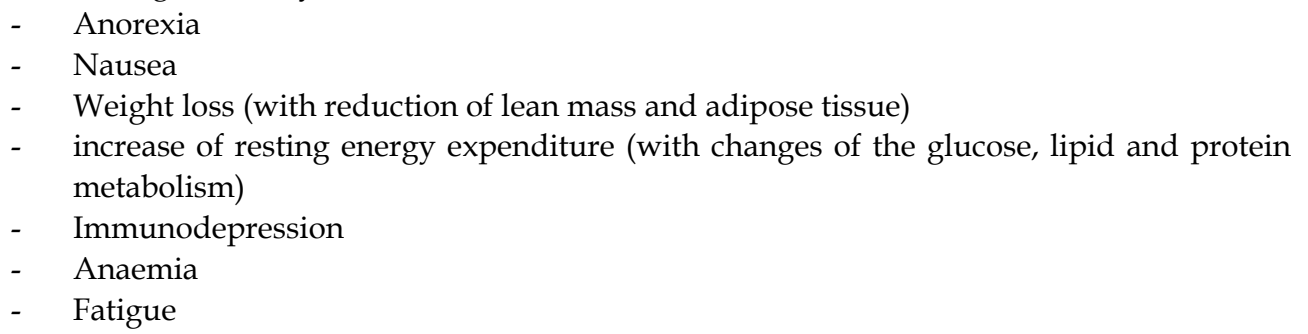

It is difficult to establish the exact moment when such changes actually start, but it could be hypothesized that they are the consequence of the interactions between the tumour and the host. The hypothesis that the presence of the tumour and its continuous growth are responsible for the increased energy expenditure and for the progressive weight loss has been considered the most reliable so far. Indeed, the presence in the host of continuously growing neoplastic tissue justifies by itself the increased energy needs; moreover, it is accompanied by enhanced energy expenditure associated with the chronic activation of the immune system, trying to counteract the tumour, which is energetically very costly $(25-30 \%$ of the basal metabolic rate, i.e. 1750-2080 kJ/day) (Straub et al., 2010). The resulting metabolic scenario is that of two systems that require a continuous supply of energy substrates, particularly glucose. Glucose oxidation to $\mathrm{CO}_{2}$ and $\mathrm{H}_{2} \mathrm{O}$ is the main energy source produced as ATP, NADH and FADH. A further glucose amount is also involved for the synthesis, through the phosphate pentose pathway, of compounds with high reducing power as NADPH and reduced glutathione (GSH), essential for the neutralisation of reactive oxygen species (ROS) produced during the various steps of the energy metabolism. ROS are intermediate compounds derived from the univalent reduction of molecular oxygen by electrons and protons, characterized by the presence of an unpaired electron in the farthest external orbital, which makes them particularly unstable (hydrogen peroxide: 
$\mathrm{H}_{2} \mathrm{O}_{2}$, superoxide anione: $\mathrm{O}_{2}$; , hydroxyl radical: $\mathrm{OH}^{\circ}$ ). As they are partly useful, but potentially toxic, compounds the body has a number of control mechanisms that limit their activity once they have been used for the scheduled objective. In particular superoxide dismutase (SOD) metabolises $\mathrm{O}_{2}^{-}$to $\mathrm{H}_{2} \mathrm{O}_{2}$, whereas catalase and various glutathione peroxidase (GPx) metabolise it to $\mathrm{H}_{2} \mathrm{O}$ and alcohol. ROS which have not been eliminated for the lack of these antioxidants, have a negative oxidative action on polyunsaturated fatty acids circulating proteins, membrane rich of disulphur bridge, enzymes and DNA, determining irreversible damage both to cell architecture and function. Under such conditions, detoxification systems, sustained by reducing compounds, should be adequately present. These reducing compounds, which are called natural detoxificants, are thus essential for a normal cell activity.

The energy metabolism in cancer patients is affected by the presence, during the disease evolution, of symptoms such as anorexia, nausea and vomiting, which prevent a normal nutrition and thus a regular supply of glucose, lipids, proteins and vitamins. Antiblastic treatments and the same molecules (cytokines), which regulate both the tumour development and the immune system functions, are responsible for these symptoms (Bennani-Baiti \& Davis, 2008). In this context, the finding that neoplastic patients in advanced stages show a severe impairment of immunologic functions characterized by impaired cell-mediated immunity and elevated serum levels of macrophage cytokines (IL-1, IL-6, TNF-a) and inflammation acute phase proteins (fibrinogen and CRP) is of great importance (Macciò et al., 1998). Evidence that high serum concentrations of cytokines and inflammatory proteins are associated with high levels of ROS and low levels of SOD and GPx is also of particular interest (Mantovani et al., 2002).

Thus, in neoplastic patients tumour growth and immune system activation determine an overall metabolic picture characterized by:

- Increased glucose, lipid and protein requirements;

- Difficulty to introduce these substances with food because of anorexia, nausea and vomiting;

- Resorting to glucogenesis with depletion of protein and lipid stores and thus loss of weight;

- Difficult to use the newly formed glucose because of hypoinsulinemia and/or peripheral resistance to insulin;

- Oxidative damage induced by ROS on DNA, membrane lipoprotein, and enzymes and coenzymes that play a major role in the regulation of the main cell anabolic and catabolic pathways.

Therefore, the metabolic changes described in the neoplastic patients are to be attributed to the chronic action of some cytokines (in particular IL-1, IL- 6 and TNF- $\alpha$ ) produced both by activated immune system and tumour cells (Argiles \& Lopez-Soriano, 1999; Delano \& Moldawer, 2006). It may be hypothesised that, during the initial phases of neoplastic disease, the synthesis of proinflammatory cytokines leads to an efficient antineoplastic effect. However, the inability of the immune system to definitively counteract tumour growth (Hagemann et al., 2006) determines the chronicisation of cytokine activity with deleterious effects on cell metabolism, body composition, nutritional status and immune system efficiency. Indeed, the chronic action of cytokines is the main cause of the metabolic abnormalities characterising advanced ovarian cancer patient (Figure 2). 
In detail, IL-1 exerts a specific effect on reducing food intake and influences meal size and duration: IL-1 has an anorectic action by directly decreasing neuropeptide Y (NPY) neurotransmission and secondarily by increasing corticotrophin-releasing factor (CRF), which in turn acts on the satiety circuitry inhibiting food intake. IL-1 has also been demonstrated to inhibit serum levels of growth hormone $(\mathrm{GH})$ by increasing CRF and somatostatin levels. The decreased synthesis of GH leads to reduced synthesis of the insulinlike growth factors (IGFs), which in turn influences the muscle protein turnover and the autocrine and paracrine regulation of muscle mass proliferation. TNF- $\alpha$ has been shown to promote lipolysis and inhibit lipogenesis and plays a key role in the depletion of adipose tissue mass seen in cachexia. It has been proposed that an elevation in plasma levels of TNF$a$ is responsible for the metabolic alterations in adipose tissue seen in advanced cancer patients. Lipid metabolism is a complex sequence of events that determine whether the triglyceride pool within the adipocyte increases, due to the processes of free fatty acid (FFA) uptake and lipogenesis, or decreases, due to the process of lipolysis. Circulating lipoproteins and triglycerides are first converted into FFA by the action of lipoprotein lipase (LPL), which is secreted by the adipocyte. FFA can then enter the adipocyte via a fatty acid transporter and, once inside the adipocyte, they are converted into the triglyceride by a multi-step-regulated enzymatic reaction, which involves acyl-CoA synthetase. In addition, triglyceride can be formed from the uptake of glucose, via glucose transporters (GLUT) 1 and 4 , into the adipocyte. The glucose can then be converted into triglyceride by the actions of a series of enzymes, which include acetyl-CoA carboxylase and fatty acid synthase. A large body of evidence now supports a role for TNF- $\alpha$ in modulating these processes. TNF- $a$ inhibits LPL activity by down-regulating its protein expression. In addition, TNF- $\alpha$ has been shown to reduce the expression of FFA transporters in adipose tissue. TNF-a could thus hinder the synthesis and entry of FFA into the adipocyte, curtailing an increase in the intracellular triglyceride pool size. Studies have also suggested that TNF-a may decrease the expression of enzymes involved in lipogenesis. Specifically, it has been suggested that acetyl-CoA carboxylase and fatty acid synthase are down regulated. Acyl-CoA synthase expression and activity have also been suggested to be down regulated by TNF- $a$. TNF- $\alpha$ has been found to promote lipolysis. TNF-a has been implicated as a factor associated with the development of insulin resistance. A positive association between plasma insulin levels and TNF- $\alpha$ mRNA from subcutaneous adipose tissue has been found in women, finding which is supported by a further study showing increased adipose TNF-a secretion in obese patients with insulin resistance. Extensive research has highlighted several potential mechanisms by which TNF- $\alpha$ induces insulin resistance. These include: accelerated lipolysis and a concomitant increase in circulating FFA concentrations, down regulation of GLUT4 synthesis, down-regulation of insulin receptor, insulin receptor substrate-1 (IRS-1) synthesis and increased Ser/Thr phosphorylation of IRS-1. Interleukin-6 is another proinflammatory cytokine with cachectic effects. The presence of tumour in mouse models was associated with early CACS and production of IL-6, whom serum levels correlated with the severity of CACS. Vice versa, the administration of anti-IL-6 antibody inhibits the comparison of CACS symptoms thus demonstrating the central pathogenetic role of this cytokine in cachectic syndrome. In vitro studies have demonstrated that IL-6 induces, similarly to IL-1, the hypothalamic release of CRF. Moreover, IL-6 acts on $\beta$ pancreatic cells similarly to IL-1 (Mantovani et al., 2001). 


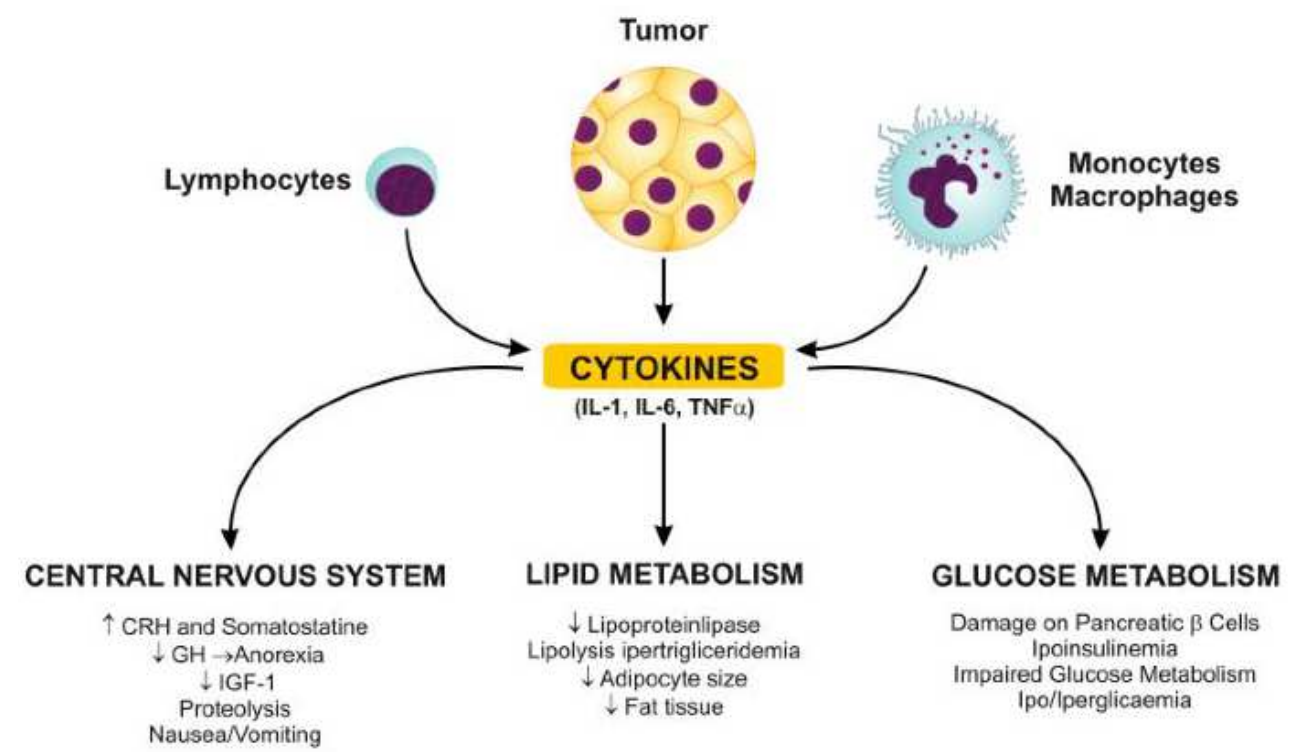

Fig. 2. Role of proinflammatory cytokines in inducing metabolic changes of advanced epithelial ovarian cancer patients. Abbreviations: IL, Interelukin; TNF, Tumour Necrosis Factor, CRH, corticotrophin releasing hormone; GH, growth hormone; IGF, Insulin growth factor.

Findings from our group demonstrated a relationship between serum levels of IL-6 and leptin, one of the most important parameters of the body energy metabolism (Macciò et al., 2008) in advanced EOC patients leptin levels were significantly lower in comparison to controls and were inversely correlated with weight, BMI, stage, PS, circulating cytokines, CRP and fibrinogen. Furthermore, multivariate regression analysis demonstrated that IL-6, besides stage of disease, was an independent predictive factor of leptin levels. These results are in accordance with those of other important studies performed on a wide population of newly diagnosed EOC patients (Mor et al., 2005; Visintin et al., 2008). Leptin, released from adipocytes into the systemic circulation proportionally to fat mass, acts as a master hormone controlling energy metabolism and weight balance. Additionally, this adipokine controls several other critical systems, including endocrine axis, bone metabolism, as well as the immune/inflammatory response. Noteworthy, our study showed that serum leptin levels evaluated in 104 ovarian cancer patients at different stage of disease (stage I-IV) were dependent both from stage of disease and serum IL-6 levels, independently of patient BMI. This finding was in contrast to the great majority of studies in cancer patients that have concluded that BMI and weight are the most important determinants of circulating leptin levels; however, it is to be noted that in the majority of these papers the impact of weight loss and the pattern of serum leptin concentration before diagnosis or study enrolment are unknown. Indeed, experimental and clinical studies have clarified that leptin production is not only strictly related to body weight and fat but it is also influenced by glucose utilization ability (Havel, 2004). Acute caloric deprivation and increased energy expenditure result in a large decrease of leptin synthesis, before major changes in body weight or fat mass have actually occurred (Chan et al., 2003). Consistently with this evidence and the findings 
obtained by some authors in tuberculosis patients (van Crevel et al., 2002), it can be suggested that the prolonged severe inflammatory response associated to the most advanced stages of EOC is responsible for the energy metabolism impairment thus downregulating and exhausting leptin production. Indeed, the stimulation of leptin synthesis by aerobic glucose metabolism is mediated through the production of ATP and through the effect of glucose oxidation on cellular redox status and pyruvate cycling. Therefore, oxidative stress, in advanced cancer patients, consequent to the low energy reserves and the inability to utilize efficiently the energy substrates, particularly glucose, may be considered the direct evidence of the metabolic impairment of which leptin is the most important parameter. Accordingly, our results demonstrated that in advanced EOC patients the lowest leptin levels and the highest IL-6 levels correlated with the highest levels of ROS and the lowest levels of GPx, the most sensitive among antioxidants to nutritional status being a selenium-dependent enzyme. In keeping with these hypotheses, our prospective study, which analyzed the changes of the above reported parameters during the course of disease in advanced EOC patients, showed that in patients who achieved objective complete response after the primary antineoplastic treatment, IL-6 levels fell to normal values and leptin increased significantly. Then, patients who achieved progression of disease (PD) showed a significant increase of IL- 6 accompanied by a significant decrease of leptin. The patients with further PD had a progressive increase of IL-6, which reached the highest concentrations in the terminal phases of disease, associated with a significant increase of CRP and fibrinogen and a further decrease of leptin. Importantly, when PD occurred leptin did not decreased proportionally to body weight that fell significantly only in the terminal phases of disease. Leptin changes strictly reflected changes of IL-6 in accordance to tumour response or disease progression (Maccio et al., 2009). It may be suggested that leptin variation reflected the changes of energy metabolism, induced by cytokines released from the tumour itself or by the aspecific activation of the immune system, even before they caused a significant body weight loss due to anorexia and muscle and fat wasting. In light of these results we can hypothesize that in EOC patients the reduced leptin production functions as a signal of increased energy expenditure and low energy reserves during the progression of the neoplastic disease. Leptin decrease in advanced EOC patients should induce an adaptative reduction of energy expenditure and an increase of appetite and food intake in response to the metabolic impairment induced by tumour growth and cancerrelated inflammation. The signal activated by the drop of leptin levels might therefore constitute the evidence of the metabolic hyperactivity of the tumour and the host immune system and the subsequent defence attempt of the host to reduce energy expenditure when energy is scarce. Leptin levels fell together with a significant weight loss, probably induced by the prolonged action of inflammatory mediators, only in the last phases of the neoplastic disease. Indeed, chronic inflammation results in severe alterations of cell metabolism, with deleterious effects on body composition, nutritional status and immune system efficiency. Therefore, IL-6 and leptin play a central role as early markers of the main metabolic alterations associated to the progression of advanced EOC, and therefore their assessment should be included in monitoring the disease outcome, especially when cancer is no longer curable with standard antineoplastic treatments and quality of life becomes the primary endpoint.

\subsection{Inflammation-related symptoms}

As widely written on, several studies have shown that inflammatory cytokines, and in particular IL-6, play a central role in the evolution of EOC and the mechanisms by which IL- 
6 may influence disease progression and outcome are extremely complex and multifactorial. In fact IL-6, as well as IL-1 and TNF-a, is responsible for symptoms such as anorexia, nausea and vomiting, weight loss and altered energy metabolism. Furthermore, high IL-6 levels are associated with an impaired efficiency of immune cells both in terms of PBMC reduced blastic response and membrane-bound IL-2 receptor expression (Macciò et al., 1998). In the same way, recent data shown that IL-6 exerts a central role in the pathogenesis of cancerrelated anaemia. Additionally, elevated serum IL-6 levels account for its endocrine activity leading to severe impairment of physical, functional and psychosocial well-being (depression, anxiety, reduced social interaction) and fatigue.

\subsubsection{Cancer-related anaemia}

Anaemia is present in more than $30 \%$ of patients with EOC at the time of initial presentation. The severity of this particular form of anaemia called cancer-related anaemia (CRA) has been associated with more aggressive tumour hystotypes and is able to influence the response to treatment and the patients' performance status (PS). The biologic and hematologic characteristics of CRA are similar to those observed in anaemia occurring in chronic inflammatory diseases. Several in vitro and in vivo studies demonstrated that high levels of proinflammatory cytokines and increased oxidative stress contribute both to the development of anaemia and to the resistance to human recombinant erythropoietin (HurEPO) (Figure 3).
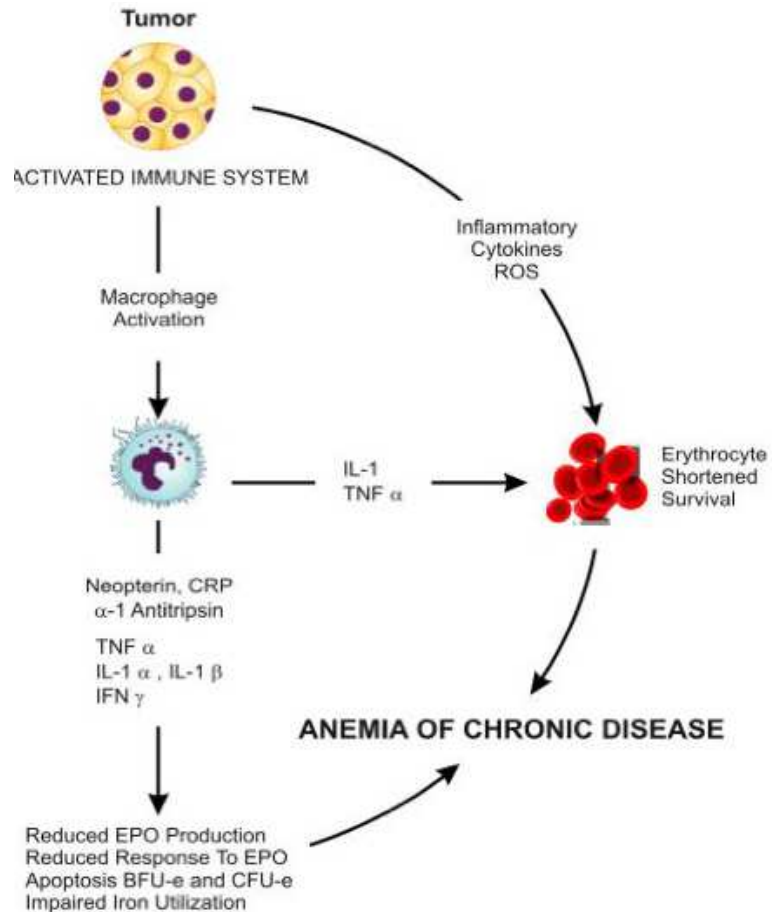

Fig. 3. Pathogenetic mechanisms of cancer-related anaemia. Abbreviations: ROS, Reactive Oxygen Species; IL, Interleukin; TNF, Tumor Necrosis Factor; IFN, Interferon; CRP, Creactive protein; EPO, erythropoietin. 
CRA is typically normochromic, normocytic with a low reticulocyte count. Bone marrow iron stores are adequate or increased, but iron reutilization is impaired, as shown by normal or increased ferritin levels and low serum iron levels and iron-binding capacity. In CRA, erythroid progenitor cells respond normally to erythropoietin (EPO), but EPO production is often not optimal for the level of anaemia. EOC patients and in particulary those in advanced stages of disease, suffer of anaemia similar to anaemia of inflammation. In these patients the lowest hemoglobin $(\mathrm{Hb})$ levels are linked with the highest concentrations of markers of inflammation, such as proinflammatory cytokines (IL-6, IL-1, TNF-a), CRP, and Fibrinogen, and with the lowest leptin levels. Statistical analysis confirmed that $\mathrm{Hb}$ inversely correlates with stage and ECOG PS, proinflammatory cytokines, CRP, Fibrinogen, and ROS but positively correlated with leptin and GPx. By multivariate regression analysis, only stage of disease and IL-6 levels are independent factors in determining Hb levels. In accordance with these data, Van der Zee et al (van der Zee et al., 1995) demonstrated that higher levels of IL-6 in cystic fluids from patients with malignant versus benign ovarian tumors correlate with decreased $\mathrm{Hb}$ levels and increased platelet counts as marker of inflammatory status. Several researchers have also demonstrated that IL-6 is both necessary and sufficient for the induction of hepcidin, an iron regulatory hormone responsible for inflammation-induced iron disutilization resulting in the anaemia associated with acute and chronic infections, chronic kidney disease, and neoplastic disease. Of note, in an our study (Macciò et al., 2005) we demonstrated a significant positive correlation between IL-6 and other markers of inflammation and oxidative stress. Thus, high serum level of IL-6 may be considered an indicator of the inflammatory and pro-oxidative status of patients with EOC and they could be linked also with a specific production of IL-6 by ovarian cancer cells. Although, it is not completely clear the mechanism through which the high levels of inflammatory mediators could induce CRA, several studies showed that proinflammatory cytokines blunt HUrEPO response to anaemia and impair erythroid colony formation in response to HUrEPO. Additionally, proinflammatory cytokines and the acute-phase proteins impair iron metabolism, inhibiting the reticuloendothelial iron stores with low iron circulating levels. Furthermore, the presence of proinflammatory cytokines in patients with EOC is associated with increased production of ROS either as a reflection of inflammation or as a consequence of their metabolic effects. Several studies demonstrated that ROSs are capable of inhibiting the production of EPO from kidney tissue. Takeda et al. (Takeda et al., 2002) hypothesized that also nutritional status, probably through leptin action, may affect erythropoiesis and demonstrated that BMI and leptin were inversely correlated with rHuEPO dose required in patients receiving hemodialysis. Indeed, in vitro studies have suggested that leptin plays a role in enhancing erythropoiesis but, certainly, this hypothesis needs more definitive analysis. Therefore, the results we have reported suggest that anaemia in patients with EOC is, at least in part, the consequence of cancer-related chronic inflammation. Cancer-related anaemia must be recognized as a constitutional feature of patients with advanced neoplasms and not necessarily as just a consequence of antineoplastic treatments. Indeed, it has been widely demonstrated that CRA is associated with poor response to treatment and decreased survival, and with a decline in energy and activity levels, quality of life, and cognitive functions. An increased understanding of the pathogenesis of CRA may help identify the most appropriate treatment strategies.

\subsubsection{Inflammation and depression}

EOC patients, who have the poorest survival rate among gynaecologic cancer patients show high rates of depression. Depression among cancer patients has frequently been attributed 
to the stress of a potentially life-threatening diagnosis and the difficulties of cancer treatment. However, several recent studies among cancer patients have found associations between depression, elevated levels of the proinflammatory cytokine and/or dysregulation of the neuroendocrine hormone cortisol (Costanzo et al., 2005). Inflammation has been implicated in the pathogenesis of depression and it has been proposed that inflammatory cytokines such as IL-6 may contribute to depression in cancer patients. Also in healthy adults, elevated IL-6 has been associated with depressive symptoms and clinical depression. In particularly IL-6 has profound effects on the CNS, inducing a syndrome of "sickness behaviors" characterized by anhaedonia and vegetative symptoms including fatigue, malaise, anorexia, difficulty concentrating, reduced activity, sleep impairments, and disinterest in activities. Proinflammatory cytokines exert differential effects on affective and vegetative depression, with more prominent effects on vegetative symptoms. Affective and vegetative depressive symptoms are thought to occur via distinct mechanisms, with vegetative symptoms occurring significantly earlier than mood disturbance. Depressive symptoms are also associated with hypercortisolemia, downregulated glucocorticoid receptors, and general dysregulation of the hypothalamic pituitary adrenocortical (HPA) axis. With chronic stress and depression, the negative feedback system regulating cortisol may become impaired and diurnal cortisol rhythms altered, particularly with respect to evening cortisol. There is a well-characterized feedback loop whereby IL-6 stimulates HPA secretion of cortisol which, in turn, exerts negative feedback on IL-6 for inflammatory control. Persistent inflammation is associated with HPA abnormalities and may contribute to the hypercortisolemia seen in depression. In particular, in advanced-stage EOC patients, assessed prior to surgery, elevations of IL- 6 associated with both affective and vegetative depressive symptoms have been documented (Lutgendorf et al., 2008). Early-stage patients had levels of IL-6 and depressive symptoms that were greater than those observed in LMP patients but lower than those in patients with advanced disease. Elevated IL-6 was also related to greater disturbances in the diurnal cortisol rhythm among advanced patients, with the elevated plasma and ascites levels of IL-6 related to higher evening cortisol as well as higher afternoon cortisol and cortisol AUC. These results are consistent with the "proinflammatory cytokine theory of depression" in suggesting that pathophysiologic elevations in circulating inflammatory mediators may lead to the appearance of depressive symptomatology via cytokine regulation of CNS function.

Proinflammatory cytokines influence the CNS via several direct pathways, including passage through permeability area of the blood-brain barrier and stimulation of afferent fibers in the vagus nerve. These fibers transmit information to specific brain nuclei with subsequent downstream effects on multiple central processes including induction of cytokines, neurotransmitters, stimulation of the HPA axis and development of sickness behaviors. Relationships between IL-6 and vegetative depression without any associations between affective depression and IL- 6 are consistent with the possibility that inflammatory mechanisms may contribute specifically to vegetative symptoms, whereas other mechanisms may underlie affective symptoms of depression. Chronic inflammation can induce glucocorticoid resistance and lead to a hyperactive HPA axis. The resultant HPA dysregulation and high levels of cortisol may contribute to depression, providing an indirect pathway linking IL-6 and depression. Then, the excessive production of IL- 6 by ovarian carcinomas may set up a chronic proinflammatory state, eliciting sickness behaviors in the CNS and hypersecretion and dysregulation of the HPA axis, both contributing to depressive symptomatology. Because of extremely high levels of tumor-secreted IL-6, particularly in 
ascites, secreted cortisol may be inadequate to suppress IL-6. In turn, depression may contribute to enhanced IL-6 secretion. In fact, depression has been associated with systemic elevations in norepinephrine which is known to enhance IL-6 secretion by ovarian tumor cells in vitro, potentially setting up a positive feedback loop for IL-6 in the tumor microenvironment. It is also possible that all of these pathways may operate simultaneously (Weinrib et al., 2010).

\subsubsection{Inflammation and fatigue}

Fatigue is one of the most common and distressing side effects of cancer and its treatment and may persist long after successful treatment completion. Subjective and objective evidence suggest that a third to half of patients developing EOC report symptoms at 3 or more months prior to diagnosis (Lurie et al., 2009; Arriba et al., 2010). Fatigue may be part of these symptom complex (Smith, 2006). Cancer-related fatigue (CRF) has been defined by National Comprehensive Cancer Network as "a distressing persistent subjective sense of tiredness or exhaustion related to cancer or cancer treatment that is not proportional to recent activity and interferes with usual functioning". It can adversely affect emotional, physical and mental well-being. CRF can also affect patients' abilities to function in terms of their usual social activities, and their ability to carry on with their normal working lives. The two most plausible mechanism include an abnormal or prolonged inflammatory response and/or disruption to the HPA axis. Emerging evidence suggests that inflammatory processes may be involved in cancer-related fatigue both during and after treatment. Indeed, a wide range of different changes of the immune system has been shown in patients suffering from fatigue. The most common are deficit of cell-mediated immunity associated with high serum levels of the proinflammatory cytokines. Each of these cytokines can determine by themselves the symptomatology typical of patient suffering from fatigue. Indeed, it is well known that these cytokines play important actions both on the central nervous system and the endocrine system and at various sites involved in the regulation of energy metabolism. The same proinflammatory cytokines involved in cachexia and associated with chronic inflammation are potent stimulators of the HPA axis. Moreover, changes in the HPA axis may be caused by a number of different factors relevant to neoplastic disease: cancer itself and/or cancer treatment can alter the function of the HPA axis resulting in endocrine changes that cause or contribute to fatigue. All these findings highlight multiple and complex mechanisms through which the immune system function disorders may lead to fatigue. Moreover, the close link between fatigue and depression in cancer patients suggests that a common mechanism could underlie the development of both. Since serotonin is a principal (but not a sole) contributor to depression, the model would predict that serotonin-influencing interventions effective against clinical depression might also prove beneficial for fatigue. Furthermore, it has been proposed that patients with cancer, particularly those with anorexia-cachexia, have altered muscle protein metabolism, which may also contribute to cancer-related fatigue.

Since the causes of fatigue are not fully understood, it is very difficult to treat it appropriately. The National Comprehensive Cancer Network's clinical guidelines also provide further options for cancer-related fatigue management. These suggest initially treating any underlying reversible causes of fatigue (e.g. anaemia, poor nutrition or depression) and attending to general supportive measures and psychosocial support. A recent review (Minton et al., 2010) has examined drug treatment for fatigue as it represents 
one of the ways this problem can be tackled. The review authors looked at trials in all types of cancer and at all stages of treatment. Fifty studies met the inclusion criteria but only 31 (7104 participants) were deemed suitable for detailed analysis as they explored fatigue in sufficient detail. They found mixed results with some drugs showing an effect on fatigue. In particular the authors concluded that Methylphenidate, a stimulant drug that improves concentration, is effective for the management of cancer-related fatigue but the small samples used in the available studies mean more research is needed to confirm its role. Erythropoietin and darbopoetin, drugs that improve anaemia, are effective in the management of cancer-related fatigue. Research on inflammation and cancer-related fatigue helps to elucidate the biological basis for this common and troublesome symptom and may also promote the development of targeted therapies. In particular, use of cytokine antagonists may be a promising direction for intervention efforts. There is preliminary evidence that TNF-a blockade with etanercept is safe and effective in reducing fatigue among patients with advanced cancer (Monk et al., 2006), but effects among patients with early stage cancer and cancer survivors have not been determined. Behavioural and mindbody interventions also show considerable promise for treating fatigue and other cancerrelated symptoms, and there is preliminary evidence for their effects on immune function (Carlson et al., 2003; Fairey et al., 2005; Stevinson et al., 2009). These treatments may be more palatable to EOC patients than pharmacologic therapies and are another important avenue for research efforts.

\subsection{Chemoresistance}

It has been shown that increased IL-6 concentration in serum and ascites of EOC patients correlates with chemoresistance. In particular, the IL-6 signalling cascade in ovarian cancer cells has been associated with the development of cisplatin and paclitaxel resistance (Wang et al, 2010). The underlining mechanisms of IL-6-mediated chemoresistance in ovarian cancer cells are not so clear. However, some studies showed that IL-6 is associated with increased expression of multidrug resistance-related genes, apoptosis inhibitory proteins (Bcl-2, Bcl-xL and XIAP) as well as activation of Ras/MEK/Erk and PI3K/Akt signalling. Moreover, IL-6 signalling prevents chemotherapy-induced endothelial cells apoptosis (Lo et al., 2011). Thus, interference with IL-6 pathway may offer opportunities for new strategies in ovarian cancer therapy. Using a monoclonal antibody that specifically blocks IL-6 signalling (siltuximab), Guo et al. demonstrated in vitro that the combination of siltuximab with paclitaxel increased the sensitivity of ovarian tumour cells to paclitaxel (Guo et al., 2010).

In vitro studies with ovarian cancer cell lines confirm that generation of paclitaxel-resistant sublines is often associated with increased IL-6 mRNA expression and protein secretion. As well known, IL-6 acts through a hexametric receptor, which contains the ligand-binding IL6a chain and the common cytokine receptor signal-transducing subunit gp130. The binding of IL-6 to gp130 activates multiple signal transduction pathways such as signal transducers and activators of transcription (JAK/STATs) pathway, Ras/MEK (mitogen-activated protein or extracellular signal-regulated kinase kinase)/ERK (extracellular signal-regulated kinase) pathway, and PI3K (phosphotidylinositol 3 kinase)/Akt pathway. Recently, evidence suggests that activation of Ras/MEK/ERK and PI3K/Akt signalling pathways play an important role in chemoresistance of EOC. A research by Wang et al (Wang et al., 2010) firstly demonstrated that autocrine production of IL-6 by ovarian cancer cell lines is 
inversely associated with their response to cisplatin and paclitaxel. Moreover, both exogenous and endogenous IL-6 induce cisplatin and paclitaxel resistance in non-IL-6producing cells, whereas deleting of endogenous IL-6 expression in IL-6-overexpressing cells promotes the sensitivity of these cells to these anticancer drugs. Meanwhile, IL-6mediated resistance of EOC cells exhibits decreased proteolytic activation of caspase- 3 and a number of studies have shown that the anti-apoptotic ability of IL-6 was associated with expression of the Bcl-2 family proteins that are typically associated with resistance to chemotherapy. Then, the main mechanism of drug resistance induced by IL- 6 is exerted in a dose dependent manner by the increased expression of Bcl-2 family proteins. Other lines of evidence suggest that also the activation of Ras/MEK/ERK and PI3K/Akt, the most important cell survival signalling, protects EOC cells from chemotherapy. It has been shown that cisplatin treatment modulates ERK and that activation of ERK protects ovarian cancer cells from cisplatin-induced death. The inhibition in vitro of ERK signalling by a MEK $1 / 2$ inhibitor blocked ERK activation and increased cisplatin sensitivity in specific EOC cell lines. Also, the inactivation of Akt and its downstream targets sensitizes human ovarian cancer cells to cisplatin and paclitaxel. Worthy of note, it is specifically IL-6 to be able to induce activation of ERK and Akt in ovarian cancer cells and that the use of specific inhibitors of these two signal transducers, inhibits IL-6-induced cisplatin and paclitaxel resistance. Taken together, these data suggest that IL-6 promotes chemoresistance of ovarian cancer cells via activation of multiple signal transduction pathways including ERK cascade and PI3K/Akt pathway. These results provide support for these signal transduction pathways as a strategy for reversing drug resistance.

Another major downstream component of the IL-6 signalling pathway is STAT3. Duan et al. (Duan et al., 2006) has reported that inhibition of STAT3 expression increases the sensitivity of ovarian cancer cell lines to paclitaxel treatment in vitro, suggesting that the STAT3 pathway may also be involved in chemoresistance of ovarian cancer cells. They found that IL-6 induced phosphorylation of STAT3 in several, but not all, of the examined ovarian cancer cell lines. However, it is possible that STAT3 could be activated also through IL-6independent mechanisms such as Src, epidermal growth factor receptor, or other cytokines like oncostatin in different cancer cells.

In conclusion, IL-6 secreted by ovarian cancer and/or immune cells may contribute to the refractoriness of these cells to conventional chemotherapy through down-regulation of various signalling step. IL-6-induced chemoresistance may be associated with increase of both multidrug resistance-related genes (MDR1 and GSTpi) and apoptosis inhibitory proteins (Bcl-2, BclxL and XIAP), as well as activation of Ras/MEK/ERK and PI3K/Akt. Then, modulation of IL-6 expression or its related signalling pathways may be a promising strategy of treatment for drug-resistant EOC.

Also COX-2 could represent a possible new marker of sensitivity to platinum-based chemotherapy in ovarian cancer. In a study by Ferrandina et al in a population of advanced ovarian cancer patients, COX-2 positivity was found in a statistically significant higher percentage of unresponsive cases than in patients responding to chemotherapy (Ferrandina et al., 2002b). The association between COX-2 positivity and poor chance of response to treatment was retained in multivariate analysis. The ability of COX-2 to predict tumour sensitivity to chemotherapy is not dependent on EGFR or Her-2/neu status and could be independently associated with prognosis. Therefore, in this context, the availability of agents able to specifically interfere with COX-2 is of potential interest. 


\section{Inflammation and possible therapeutic implications}

Our knowledge on ovarian cancer-related inflammation offers innovative therapeutic strategies. For many years, all efforts to treat cancer have concentrated on the destruction/inhibition of tumour cells. Strategies to modulate the host microenvironment offer a complementary perspective. Primary proinflammatory cytokines represent the main targets and ongoing results in this direction justify continuing efforts (Colotta et al., 2009).

In particular, IL-6, as described above, plays a central role in EOC in promoting tumour growth and progression and influencing its prognosis and related symptoms. Collectively, all data available in the literature and reported in the previous sections of this chapter lead to hypothesize that IL-6 antagonists may have therapeutic activity in patients with ovarian cancer via inhibition of a tumour-promoting cytokine network. Accordingly to this evidence, Coward et al. (Coward et al., 2011) carried out an experimental study to assess the activity of the anti-human-IL-6 antibody siltuximab (CNTO328) in tissue culture of EOC and human ovarian cancer xenografts. The authors demonstrated that IL-6 is expressed both in malignant cells and infiltrating leukocytes, endothelial cells and stromal fibroblast. In addition, they found that high IL-6 expression in EOC cells was associated with poor prognosis. Vice versa, IL-6 inhibition prevents the constitutive production of IL-6 and other inflammatory and angiogenic mediators by EOC cells. Additionally, siltuximab had also a significant inhibitory effect on tumour cell proliferation, macrophage infiltration and angiogenesis. In the same paper Coward et al presented the results of a single arm phase II clinical trial of the anti-human IL-6 monoclonal antibody siltuximab in women with recurrent ovarian cancer. Interestingly, they showed that siltuximab, given as a single agent, has some clinical activity in recurrent, platinum-resistant ovarian cancer. A total of eight patients achieved radiological disease stabilisation, which lasted six months or more in four cases. One of these eight also had normalisation of CA125 that lasted for 12 weeks, giving an overall partial response by combined RECIST/CA125 criteria. Noteworthy, partial response was accompanied by a reduction in ${ }^{18} \mathrm{~F}$ FDG uptake as detected by PET/TC imaging. Moreover, siltuximab treatment induced a decline in plasma levels of CRP, CCL2, CXCL12, VEGF and IL-8. Also a significant increase in $\mathrm{Hb}$ levels occurred in the majority of patients. The study by Coward et al is the first clinical study of anti-IL-6 therapy carried out in a population of EOC patients. Several experimental studies support the rationale for using this anti-IL-6 mAb in EOC. In fact, it has been demonstrated that siltuximab specifically suppress IL-6-induced STAT3 phosphorylation and STAT3 nuclear translocation, as well as the levels of Stat3 downstream proteins such as MCL-1, Bcl-X (L), and surviving, thus targeting the main intracellular mediator of the effects of cytokines on EOC cells growth (Guo et al., 2010).

Indeed, as well described above, STAT3 is constitutively active in EOC and leads to increased expression of genes regulating survival and proliferation, and drives the malignant behaviour of these cells. Therefore, the identification of novel compounds that selectively inhibit STAT3 activity may lead to additional useful tools to reduce cancerassociated cell proliferation, inflammation, and chemotherapeutic resistance. A potent and selective STAT3 inhibitor has been identified through the use of high throughput screening, synthetic medicinal chemistry, and molecular assays. Due to the central role of aberrant STAT3 signalling in ovarian cancer pathogenesis, this compound may provide a useful starting point for the development of chemical scaffolds to block STAT3 signalling for cancer therapy (Madoux et al., 2010). In particular, STAT3 dimerization inhibitors 
could play a significant role in the future of cancer and adjuvant cancer therapies (Lavecchia et al., 2011).

STAT3 activation is also induced by hypoxia that is commonly observed in many solid tumours and represents a major obstacle to chemo- or radiation therapy. In an experimental animal model it has been shown that exposure of mice containing human ovarian cancer xenograft tumour to hyperbaric oxygen (HBO) obtained a significant reduction in tumour volume, associated with a significant decrease of STAT3 (Tyr 705) activation and cyclin-D1 protein/mRNA levels. Interestingly, $\mathrm{HBO}$ exposure, in combination with weekly administration of cisplatin, also significantly reduced the tumour volume. Therefore, therapeutic strategies able to increase tumour oxygenation may be able to inhibit key steps, such as STAT3 activation, involved in the ovarian tumour progression. (Selvendiran et al., 2010). Moreover, the reduced effectiveness of conventional chemotherapeutic drugs cisplatin and taxol in eliminating the hypoxic ovarian cancer cells suggests a role for pSTAT3 in cellular resistance to chemotherapy. It has been shown that inhibition of STAT3 followed by treatment with cisplatin or taxol resulted in a significant increase in apoptosis supporting the hypothesis that hypoxia-induced STAT3 activation is responsible for chemoresistance (Selvendiran et al., 2009). According to this evidence the correction of anaemia and the maintenance of adequate $\mathrm{Hb}$ levels during cancer chemotherapy should be addressed as a fundamental outcome in the therapeutic strategies of EOC.

Disruption of STAT3 could also be therefore an effective approach to control EOC tumorigenesis. Among the several compounds tested for chemoprevention of EOC curcumin is one of the most interesting and studied. Curcumin is a dihydroxyphenolic compound, whose anti-tumour mechanisms involve regulation of STAT-3 and the negative regulators of STAT-3, including suppressors of cytokine signalling proteins (SOCS-1 and SOCS-3), protein inhibitors of activated STAT (PIAS-1 and PIAS-3), and SH2 domaincontaining phosphatases (SHP-1 and SHP-2). Treatment of ovarian cancer cells with curcumin induced a dose- and time-dependent decrease of constitutive IL-6 expression and IL-6-induced STAT-3 phosphorylation, which is associated with decreased cell viability and increased cleavage of caspase-3. Moreover, curcumin suppresses JAK-STAT signalling also via activation of PIAS-3, thus attenuating STAT-3 phosphorylation and tumour cell growth (Saydmohammed et al., 2010). The activity of curcumin on STAT3 is also mediated by its ability to inhibit lysophosphatidic acid (LPA) which is a biolipid that stimulates tumour cell invasion and metastasis by inducing phosphorylation of STAT3 as well as IL-6 and IL-8 secretion, which in turn results in STAT3 phosphorylation. Treatment of the cells with curcumin inhibited LPA-induced IL-6 and IL-8 secretion and STAT3 phosphorylation, leading to blocked ovarian cancer cell motility (Seo et al., 2010).

Since the same inflammatory mediators that promote tumour growth also are responsible for cancer-related symptoms, i.e., cachexia/anorexia, anaemia, fatigue, pain, debilitation and shortened survival, a concerted effort should be made to attack inflammation alongside with other anticancer measures at initial diagnosis with the consequent probability of improving both patient quality of life and survival (MacDonald, 2007). Therefore, counteracting cancerrelated inflammation is certainly a key target in the therapeutic approach of symptoms associated to advanced cancer, especially in EOC patients who are diagnosed at advanced stage and suffer of severe distressing symptoms. A suggestive example of how the 
modulation of inflammation may be useful in the care of EOC patients is represented by the efficacy of lactoferrin in association to rHuEPO in the treatment of chemotherapy-induced anaemia. In fact, lactoferrin is a specific protein involved in iron transport mechanisms, which has also an important role in host defence against infection and excessive inflammation. Results from a recent open label randomised phase III study of our group (Macciò et al., 2010), including EOC patients (20\% in each arm), demonstrated that lactoferrin plus rHuEPO was able to increase $\mathrm{Hb}$ levels with a efficacy similar to iron i.v. in term of haematopoietic response but with a better capacity to modulate iron homeostasis and inflammation (as demonstrated by decrease of ferritin and CRP levels in patients treated with lactoferrin).

Specific inhibition of proinflammatory cytokines, and particularly IL-6, has also been tested in the therapeutic approach of cancer-related cachexia. Preliminary results form a phase I study showed that i.v. infusion of a specific anti-IL-6 MoAb was able to reverse fatigue, increase haemoglobin and albumin, and improve muscle strength (Clarke et al., 2009). However, according to the most recent findings, the best management of cancer-related symptoms, such as weight loss, muscle wasting, anorexia, anaemia, fatigue, which globally define the clinical picture of cachexia, requires a multimodal approach by a multidisciplinary team and is best commenced earlier rather than later (Bosaeus, 2008). Intervention should include dietary counselling, nutritional and vitamin supplementation, exercise concordant with the patient's physical condition, anti-inflammatory agents, anabolic drugs and the most adequate symptom managements.

In the context of such combined approaches, one of the most intriguing ones was an open phase II trial published by our group (Mantovani et al., 2006) which aimed to test the safety and efficacy of an integrated treatment based on diet, pharmaconutritional support administered orally, and drugs in a population of cachectic patients with advanced cancer at different sites, including also a significant percentage of EOC patients. The treatment consisted of diet with high polyphenols content $(400 \mathrm{mg})$, antioxidant treatment $(300 \mathrm{mg} /$ day alpha lipoic acid $+2.7 \mathrm{~g} /$ day carbocysteine lysine salt $+400 \mathrm{mg} /$ day vitamin $\mathrm{E}+30,000 \mathrm{IU} /$ day vitamin A+500 mg/day vitamin C), and pharmaconutritional support enriched with two cans per day (n-3)-PUFA (eicosapentaenoic acid and docosahexaenoic acid), $500 \mathrm{mg} /$ day MPA and 200 $\mathrm{mg} /$ day selective cyclooxygenase- 2 inhibitor celecoxib. The treatment duration was 4 months. Body weight increased significantly from baseline, as did LBM and appetite. There was an important decrease of proinflammatory cytokines IL- 6 and TNF-a, and a negative relationship worthy of note was found between LBM and IL-6 changes. As for quality of life, there was a significant improvement in the European Organization for Research and Treatment of Cancer (EORTC) QLQ-C30, Euro QL-5D and fatigue assessed by Multidimensional Fatigue Symptom Inventory-Short Form (MFSI-SF) scores. The results overall showed the treatment to be both safe (without significant adverse events) and effective as for increase of body weight, increase of LMB, decrease of proinflammatory cytokines, improvement of quality of life parameters, amelioration of fatigue symptom. On the basis of these results, we started a phase III randomized clinical trial (Mantovani et al., 2010) to establish which was the most effective and safest treatment of CACS and oxidative stress in improving selected key variables as primary endpoints: increase of LBM, decrease of REE, increase of total daily physical activity, decrease of IL- 6 and TNF-a, and improvement of fatigue. Three hundred thirty-two assessable patients with cancer-related anorexia/cachexia syndrome, including a significant 
proportion of advanced EOC patients, were enrolled. All patients were given as basic treatment polyphenols plus antioxidant agents alpha-lipoic acid, carbocysteine, and vitamins $\mathrm{A}, \mathrm{C}$, and $\mathrm{E}$, all orally administered. Then patients were randomly assigned to one of five treatment arms: arm 1, MPA (500 mg/day) or MA (320 mg/day); arm 2, oral supplementation with EPA; arm 3, L-carnitine (4 g/day); arm 4, thalidomide ( $200 \mathrm{mg} /$ day); and arm 5, a combination of the above (Figure 4).

Treatment duration was 4 months. Analysis of variance showed a significant difference between treatment arms. A post hoc analysis showed the superiority of arm 5 over the others for all primary endpoints. An analysis of changes from baseline showed that LBM (by dual-energy X-ray absorptiometry and by L3 computed tomography) significantly increased in arm 5. REE decreased significantly and fatigue improved significantly in arm 5. Appetite increased significantly in arm 5; IL-6 decreased significantly in arm 5 and arm 4; Glasgow Prognostic Score (GPS) and Eastern Cooperative Oncology Group (ECOG) performance status (PS) score decreased significantly in arm 5, arm 4, and arm 3. Toxicity was quite negligible, and was comparable between arms. In conclusion, the most effective treatment in terms of all three primary efficacy endpoints and the secondary endpoints appetite, IL-6, GPS, and ECOG PS score was the combination regimen that included all selected agents.

\section{TREATMENT PLAN}

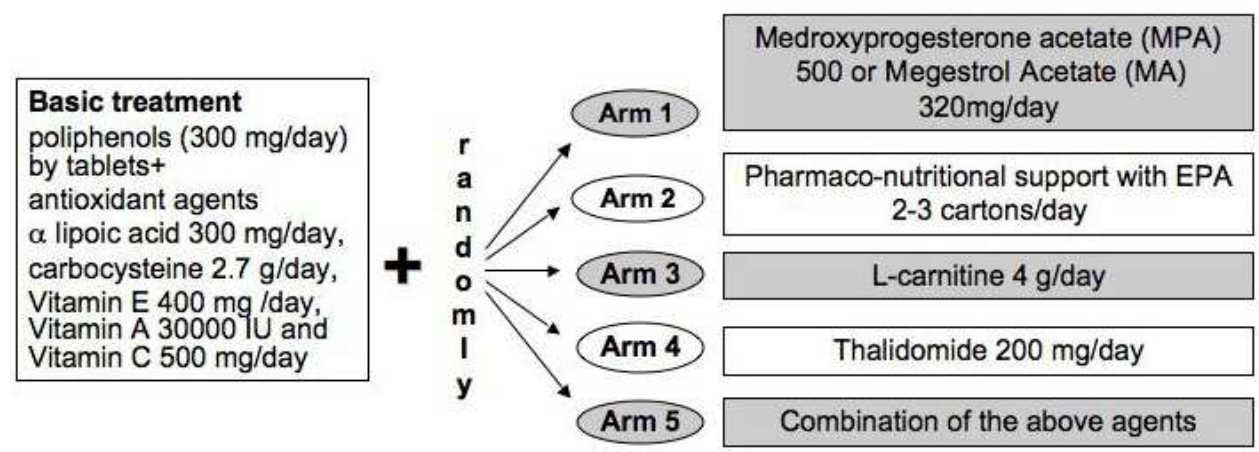

Fig. 4. Phase III randomised clinical trial of five different arms of treatment for cancer cachexia: treatment plan.

\section{Conclusion}

Proinflammatory cytokines, and in particular IL-6, as demonstrated in the present chapter, are involved in the development and progression of EOC. They are also associated with fatigue, depression, anaemia, pain and cachexia that impact significantly quality of life. Strategies to inhibit the effect of inflammation and such cytokines might therefore have a profound effect on quality of life and survival. In particular, IL-6 antagonism seems to have the most promising therapeutic activity in EOC patients but further clinical trials testing it 
both alone and in a multimodal approach are warranted. Certainly, from the body of evidence described in this chapter, it is clear that the assessment of inflammation markers, and especially IL-6, should be included in monitoring EOC during its course, from diagnosis to terminal stages, in order to develop the most appropriate care of EOC patients and allow the best supportive therapy considered as the irreplaceable therapeutic approach concurring to patients global well-being.

\section{References}

Ali-Fehmi, R., Semaan, A., Sethi, S., Arabi, H., Bandyopadhyay, S., Hussein, Y.R., Diamond, M.P., Saed, G., Morris, R.T., \& Munkarah, A.R. (2011). Molecular typing of epithelial ovarian carcinomas using inflammatory markers. Cancer Vol. 117, No. 2 (January 2011), pp. 301-309

Altinoz MA, \& Korkmaz R. (2004). NF-kappaB, macrophage migration inhibitory factor and cyclooxygenase-inhibitions as likely mechanisms behind the acetaminophen- and NSAID-prevention of the ovarian cancer. Neoplasma Vol. 51, No. 4, pp. 239-247

Argilés, J.M., \& López-Soriano, F.J. The role of cytokines in cancer cachexia. Medicinal Research Reviews Vol. 19, No. 3 (May 1999), pp. 223-248

Arriba, L.N., Fader, A.N., Frasure, H.E., \& von Gruenigen, V.E. (2010). A review of issues surrounding quality of life among women with ovarian cancer. Gynecologic Oncology Vol. 119, No. 2 (November 2010), pp. 390-396

Bast, R.C. Jr, Boyer, C.M., Jacobs, I., Xu, F.J., Wu, S., Wiener, J., Kohler, M., \& Berchuck, A. (1993). Cell growth regulation in epithelial ovarian cancer. Cancer Vol. 71, no. 4 Suppl (February 1993), pp. 1597-1601

Bennani-Baiti, N., \& Davis MP. (2008). Cytokines and cancer anorexia cachexia syndrome. The American Journal of Hospice \& Palliative Care Vol. 25, No. 5 (October-November 2008), pp. 407-411

Bosaeus, I. (2008). Nutritional support in multimodal therapy for cancer cachexia. Support Care Cancer Vol. 16, No. 5 (May 2008), pp. 447-451

Carlson, L.E., Speca, M., Patel, K.D., \& Goodey, E. (2003). Mindfulness-based stress reduction in relation to quality of life, mood, symptoms of stress, and immune parameters in breast and prostate cancer outpatients. Psychosomatic Medicine Vol. 65, No. 4 (July-August 2003), pp. 571-581

Chan, J.L., Heist, K., DePaoli, A.M., Veldhuis, J.D., \& Mantzoros, C.S. (2003). The role of falling leptin levels in the neuroendocrine and metabolic adaptation to short-term starvation in healthy men. Journal of Clinical Investigation Vol. 111, No. 9 (May 2003), pp. 1409-1421

Clarke SJ, Smith JT, Gebbie C, Sweeney, C., \& Olszewski, N. (2009). A phase I, pharmacokinetic (PK), and preliminary efficacy assessment of ALD518, a humanized anti-IL-6 antibody, in patients with advanced cancer humanized antiIL-6 antibody, in patients with advanced cancer. J Clin Oncol, Vol. 27, No. 15s (suppl), abstr 3025.

Clendenen, T.V., Lundin, E., Zeleniuch-Jacquotte, A., Koenig, K.L., Berrino, F., Lukanova, A., Lokshin, A.E., Idahl, A., Ohlson, N., Hallmans, G., Krogh, V., Sieri, S., Muti, P., Marrangoni, A., Nolen, B.M., Liu, M., Shore, R.E., \& Arslan, A.A. (2011). Circulating inflammation markers and risk of epithelial ovarian cancer. Cancer epidemiology, biomarkers E prevention 2011 Vol. 20, No. 5 (May 2011), pp. 799-810 
Colomiere, M., Ward, A.C., Riley, C., Trenerry, M.K., Cameron-Smith, D., Findlay, J., Ackland, L., \& Ahmed, N. (2009). Cross talk of signals between EGFR and IL-6R through JAK2/STAT3 mediate epithelial-mesenchymal transition in ovarian carcinomas. British Journal of Cancer Vol. 100, No. 1 (January 2009), pp. 134-144

Colotta, F., Allavena, P., Sica, A., Garlanda, C., \& Mantovani, A. (2009). Cancer-related inflammation, the seventh hallmark of cancer: links to genetic instability. Carcinogenesis Vol. 30, No. 7 (July 2009), pp. 1073-1081

Costanzo, E.S., Lutgendorf, S.K., Sood, A.K., Anderson, B., Sorosky, J., \& Lubaroff, D.M. (2005). Psychosocial factors and interleukin-6 among women with advanced ovarian cancer. Cancer Vol. 104, No. 2 (July 2005), pp. 305-313

Coward, J., Kulbe, H., Chakravarty, P., Leader, D.A., Vassileva, V., Leinster, D.A., Thompson, R., Schioppa, T., Nemeth, J.A., Vermeulin, J., Singh, N., Avril, N.E., Cummings, J., Rexhepaj, E., Jirstrom, K., Gallagher, W.M., Brennan, D.J., McNeish, I.A., \& Balkwill, F.R. (2011). Interleukin-6 as a therapeutic target in human ovarian cancer. Clin Cancer Res. 2011 Jul 27.

Cubillos-Ruiz, J.R., Rutkowski, M., \& Conejo-Garcia, J.R. (2010). Blocking ovarian cancer progression by targeting tumor microenvironmental leukocytes. Cell Cycle Vol. 9, No. 2 (January 2010), pp. 260-268

Curiel., T.J., Coukos, G., Zou, L., Alvarez, X., Cheng, P., Mottram, P., Evdemon-Hogan, M., Conejo-Garcia, J.R., Zhang, L., Burow, M., Zhu, Y., Wei, S., Kryczek, I., Daniel, B., Gordon, A., Myers, L., Lackner, A., Disis, M.L., Knutson, K.L., Chen, L., \& Zou, W. (2004). Specific recruitment of regulatory $\mathrm{T}$ cells in ovarian carcinoma fosters immune privilege and predicts reduced survival. Nature Medicine Vol 10, No. 9 (September 2004), pp. 942-949

Delano, M.J., \& Moldawer, L,L. (2006). The origins of cachexia in acute and chronic inflammatory diseases. Nutr Clin Pract Vol. 21, No. 1 (February 2006), pp. 68-81

Duan, Z., Foster, R., Bell, D.A., Mahoney, J., Wolak, K., Vaidya, A., Hampel, C., Lee, H., \& Seiden, M,V. (2006). Signal transducers and activators of transcription 3 pathway activation in drug-resistant ovarian cancer. Clin Cancer Res Vol. 12, No. 17 (September 2006), pp. 5055-5063

Fairey, A.S., Courneya, K.S., Field, C.J., Bell, G.J., Jones, L.W., \& Mackey, J.R. (2005). Randomized controlled trial of exercise and blood immune function in postmenopausal breast cancer survivors. J Appl Physiol Vol. 98, No. 4 (April 2005), pp. 1534-1540

Ferrandina, G., Ranelletti, F.O., Lauriola, L., Fanfani, F., Legge, F., Mottolese, M., Nicotra, M.R., Natali, P.G., Zakut, V.H., \& Scambia, G. (2002). Cyclooxygenase-2 (COX-2), epidermal growth factor receptor (EGFR), and Her-2/neu expression in ovarian cancer. Gynecologic Oncology Vol. 85, No. 2 (May 2002), pp. 305-310

Ferrandina, G., Lauriola, L., Zannoni, G.F., Fagotti, A., Fanfani, F., Legge, F., Maggiano, N., Gessi, M., Mancuso, S., Ranelletti, F.O., \& Scambia, G. (2002). Increased cyclooxygenase-2 (COX-2) expression is associated with chemotherapy resistance and outcome in ovarian cancer patients. Annals of Oncology Vol 13, No. 8 (August 2002), pp. 1205-1211

Gavalas, N.G., Karadimou, A., Dimopoulos, M.A., \& Bamias, A. (2010). Immune response in ovarian cancer: how is the immune system involved in prognosis and therapy: potential for treatment utilization. Clinical \& Developmental Immunology Vol. 2010 (January 2011), pp. 791603. 
Giuntoli, R.L. 2nd, Webb, T.J., Zoso, A., Rogers, O., Diaz-Montes, T.P., Bristow, R.E., \& Oelke, M. (2009). Ovarian cancer-associated ascites demonstrates altered immune environment: implications for antitumor immunity. Anticancer Research Vol. 29, No. 8 (August 2009), pp. 2875-2884

Hagemann, T., Wilson, J., Burke, F., Kulbe, H., Li, N.F., Plüddemann, A., Charles, K., Gordon, S., \& Balkwill, F.R. (2006). Ovarian cancer cells polarize macrophages toward a tumorassociated phenotype. J Immunol Vol. 176, No. 8 (April 2006), pp. 5023-5032

Havel, P.J. (2004). Update on adipocyte hormones: regulation of energy balance and carbohydrate/lipid metabolism. Diabetes Vol. 53, Suppl. 1 (February 2004), pp. S143-S151

Jeannin, P., Duluc, D., \& Delneste, Y. (2011). IL-6 and leukemia-inhibitory factor are involved in the generation of tumor-associated macrophage: regulation by IFN-ץ. Immunotherapy Vol. 3, No. 4 Suppl (April 2011), pp. 23-26

Kim, S., Hagemann, A., \& DeMichele, A. (2009). Immuno-modulatory gene polymorphisms and outcome in breast and ovarian cancer. Immunological Investigations Vol. 38, No. 3-4, pp. 324-340

Landen, C.N. Jr., Birrer, M.J., \& Sood, A.K. (2008). Early events in the pathogenesis of epithelial ovarian cancer. Journal of Clinical Oncology Vol. 26, No. 6 (February 2008), pp. 995-1005

Lane, D., Matte, I., Rancourt, C., \& Piché, A. (2011). Prognostic significance of IL-6 and IL-8 ascites levels in ovarian cancer patients. BMC Cancer Vol. 11 (May 2011), pp. 210

Lavecchia, A., Di Giovanni, C., \& Novellino, E. (2011). STAT-3 inhibitors: state of the art and new horizons for cancer treatment. Current Medicinal Chemistry Vol 18, No. 16, pp. 2359-2375

Leffers, N., Gooden, M.J., de Jong, R.A., Hoogeboom, B.N., ten Hoor, K.A., Hollema, H., Boezen, H.M., van der Zee, A.G., Daemen, T., \& Nijman, H.W. (2009). Prognostic significance of tumor-infiltrating T-lymphocytes in primary and metastatic lesions of advanced stage ovarian cancer. Cancer Immunol Immunother Vol. 58, No. 3 (March 2009), pp. 449-459

Liao, S., Liu, J., Lin, P., Shi, T., Jain, R.K., \& Xu, L. (2011). TGF-beta blockade controls ascites by preventing abnormalization of lymphatic vessels in orthotopic human ovarian carcinoma models. Clin Cancer Res Vol. 17, No. 6 (March 2011), pp. 1415-1424

Lo, C.W., Chen, M.W., Hsiao, M., Wang, S., Chen, C.A., Hsiao, S.M., Chang, J.S., Lai, T.C., Rose-John, S., Kuo, M.L., \& Wei, L.H. (2011). IL-6 trans-signaling in formation and progression of malignant ascites in ovarian cancer. Cancer Research Vol. 71, No. 2 (Jan 2011), pp. 424-434

Lurie, G., Thompson, P.J., McDuffie, K.E., Carney, M.E., \& Goodman MT. (2009). Prediagnostic symptoms of ovarian carcinoma: a case-control study. Gynecologic Oncology Vol. 114, No. 2 (August 2009), pp. 231-236

Lutgendorf, S.K., Weinrib, A.Z., Penedo, F., Russell, D., DeGeest, K., Costanzo, E.S., Henderson, P.J., Sephton, S.E., Rohleder, N., Lucci, J.A. 3'rd, Cole, S., Sood, A.K., \& Lubaroff, D.M. (2008). Interleukin-6, cortisol, and depressive symptoms in ovarian cancer patients. J Clin Oncol Vol. 26, No. 29 (October 2008), pp. 4820-4827

Macciò, A., Mantovani, G., Turnu, E., Artini, P., Contu, G., \& Volpe, A. (1994). Preovulatory human follicular fluid in vitro inhibits interleukin (IL)-1 alpha, IL-2, and production and expression of p55 chain IL-2 receptor of lymphomonocytes. Fertility and Sterility Vol. 62, No. 2 (August 1994), pp. 327-332 
Macciò, A., Lai, P., Santona, M.C., Pagliara, L., Melis, G.B., \& Mantovani, G. (1998). High serum levels of soluble IL-2 receptor, cytokines, and $C$ reactive protein correlate with impairment of $\mathrm{T}$ cell response in patients with advanced epithelial ovarian cancer. Gynecology Oncology Vol 69, No. 3 (June 1998), pp. 248-252

Macciò, A., Madeddu, C., Massa, D., Mudu, M.C., Lusso, M.R., Gramignano, G., Serpe, R., Melis, G.B., \& Mantovani G. (2005). Hemoglobin levels correlate with interleukin-6 levels in patients with advanced untreated epithelial ovarian cancer: role of inflammation in cancer-related anemia. Blood Vol. 106, No. 1 (July 2005), pp. 362-367

Macciò, A., Madeddu, C., Massa, D., Astara, G., Farci, D., Melis, G.B., \& Mantovani, G. (2009). Interleukin-6 and leptin as markers of energy metabolic changes in advanced ovarian cancer patients. Journal of Cellular and Molecular Medicine Vol. 13, No. 9B (September 2009), pp. 3951-3959

Macciò, A., Madeddu, C., Gramignano, G., Mulas, C., Sanna, E., \& Mantovani, G. (2010). Efficacy and safety of oral lactoferrin supplementation in combination with rHuEPO-beta for the treatment of anemia in advanced cancer patients undergoing chemotherapy: open-label, randomized controlled study. Oncologist Vol. 15, No. 8 (July 2010), pp. 894-902

MacDonald, N. (2007). Cancer cachexia and targeting chronic inflammation: a unified approach to cancer treatment and palliative/supportive care. The Journal of Supportive Oncology Vol. 5, No. 4 (April 2007), pp. 157-162

Madoux, F., Koenig, M., Sessions, H., Nelson, E., Mercer, B.A., Cameron, M., Roush, W., Frank, D., \& Hodder, P. (2010). Modulators of STAT Transcription Factors for the Targeted Therapy of Cancer (STAT3 Inhibitors). Probe Reports from the NIH Molecular Libraries Program [Internet]. Bethesda (MD): National Center for Biotechnology Information (US) (August 2009) updated 2011 Mar 25

Mantovani, G., Macciò, A., Pisano, M., Versace, R., Lai, P., Esu, S., Massa, E., Ghiani, M., Dessì, D., Melis, G.B., \& Del Giacco, G.S. (1997). Tumor-associated lympho-monocytes from neoplastic effusions are immunologically defective in comparison with patient autologous PBMCs but are capable of releasing high amounts of various cytokines. International Journal of Cancer Vol. 71, No. 5 (May 1997), pp. 724-731

Mantovani, G., Macciò, A., Massa, E., Lai, P., Manca, G., Mudu, C., Versace, R., \& Pusceddu G. (1999). Relationships betxween Fas expression, activation molecule CD25, and functional activity of tumor-associated lymphomonocytes from neoplastic effusions. Oncology Reports Vol. 6, No. 1 (January-February 1999), pp. 235-239

Mantovani, G., Macciò, A., Melis, G., Mura, L., Massa, E., \& Mudu, M.C. (2000). Restoration of functional defects in peripheral blood mononuclear cells isolated from cancer patients by thiol antioxidants alpha-lipoic acid and $\mathrm{N}$-acetyl cysteine. Int J Cancer Vol 86, No. 6 (June 2000), pp. 842-847

Mantovani, G., Macciò, A., Massa, E., \& Madeddu C. (2001). Managing cancer-related anorexia/cachexia. Drugs Vol. 61, No. 4, pp. 499-514

Mantovani, G., Macciò, A., Madeddu, C., Mura, L., Gramignano, G., Lusso, M.R., Mulas, C., Mudu, M.C., Murgia, V., Camboni, P., Massa, E., Ferreli, L., Contu, P., Rinaldi, A., Sanjust, E., Atzei, D., \& Elsener, B. (2002). Quantitative evaluation of oxidative stress, chronic inflammatory indices and leptin in cancer patients: correlation with stage and performance status. International Journal of Cancer Vol. 98, No. 1 (March 2002), pp. 84-91

Mantovani, G., Macciò, A., Madeddu, C., Mura, L., Gramignano, G., Lusso, M.R., Massa, E., Mocci, M., \& Serpe, R. (2003). Antioxidant agents are effective in inducing 
lymphocyte progression through cell cycle in advanced cancer patients: assessment of the most important laboratory indexes of cachexia and oxidative stress. $\mathrm{J} \mathrm{Mol}$ Med (Berl) Vol. 81, No. 10 (October 2003), pp. 664-73

Mantovani, G., Macciò, A., Madeddu, C., Gramignano, G., Lusso, M.R., Serpe, R., Massa, E., Astara, G., \& Deiana, L. (2006). A phase II study with antioxidants, both in the diet and supplemented, pharmaconutritional support, progestagen, and anticyclooxygenase-2 showing efficacy and safety in patients with cancer-related anorexia/cachexia and oxidative stress. Cancer Epidemiology Biomarkers \& Prevention Vol. 15, No. 5 (May 2006), pp. 1030-1034

Mantovani, G., Macciò, A., Madeddu, C., Serpe, R., Massa, E., Dessì, M., Panzone, F., \& Contu, P. (2010). Randomized phase III clinical trial of five different arms of treatment in 332 patients with cancer cachexia. Oncologist Vol. 15, No. 2 (February 2010), pp. 200-211.

Min, H., \& Wei-hong, Z. (2009). Constitutive activation of signal transducer and activator of transcription 3 in epithelial ovarian carcinoma. The journal of obstetrics and gynaecology research Vol 35, No. 5 (October 2009), pp. 918-925

Minton, O., Richardson, A., Sharpe, M., Hotopf, M., \& Stone, P. (2010). Drug therapy for the management of cancer-related fatigue. Cochrane Database Syst Rev 2010 Jul 7;(7):CD006704.

Monk, J.P., Phillips, G., Waite, R., Kuhn, J., Schaaf, L.J., Otterson, G.A., Guttridge, D., Rhoades, C., Shah, M., Criswell, T., Caligiuri, M.A., \& Villalona-Calero, M.A. (2006). Assessment of tumor necrosis factor alpha blockade as an intervention to improve tolerability of dose-intensive chemotherapy in cancer patients. J Clin Oncol Vol. 24, No. 12 (April 2006), pp. 1852-1859

Mor, G., Visintin, I., Lai, Y., Zhao, H., Schwartz, P., Rutherford, T., Yue, L., Bray-Ward, P., Ward, D.C. (2005). Serum protein markers for early detection of ovarian cancer. Proc Natl Acad Sci U S A Vol. 102, No. 21 (May 2005), pp. 7677-7682

Napoletano C, Bellati F, Landi R, Pauselli S, Marchetti C, Visconti V, Sale P, Liberati M, Rughetti A, Frati L, Panici PB, Nuti M. Ovarian cancer cytoreduction induces changes in T cell population subsets reducing immunosuppression. J Cell Mol Med. 2010 Dec;14(12):2748-59. doi: 10.1111/j.1582-4934.2009.00911.x.

Nowak, M., Glowacka, E., Szpakowski, M., Szyllo, K., Malinowski, A., Kulig, A., Tchorzewski, H., \& Wilczynski, J. (2010). Proinflammatory and immunosuppressive serum, ascites and cyst fluid cytokines in patients with early and advanced ovarian cancer and benign ovarian tumors. Neuro Endocrinology Letters, Vol. 31, No. 3, pp. 375-83

Nowak, M., Klink, M., Glowacka, E., Sulowska, Z., Kulig, A., Szpakowski, M., Szyllo, K., \& Tchorzewski, H. (2010). Production of cytokines during interaction of peripheral blood mononuclear cells with autologous ovarian cancer cells or benign ovarian tumour cells. Scand J Immunol Vol 71, No. 2 (February 2010), pp. 91-98

Plante, M., Rubin, S.C., Wong, G.Y., Federici, M.G., Finstad, C.L., \& Gastl, G.A. (1994). Interleukin-6 level in serum and ascites as a prognostic factor in patients with epithelial ovarian cancer. Cancer Vol. 73, No. 7 (April 1994), pp. 1882-1888

Rabinovich, A., Medina, L., Piura, B., Segal, S., \& Huleihel, M. (2007). Regulation of ovarian carcinoma SKOV-3 cell proliferation and secretion of MMPs by autocrine IL-6. Anticancer Research Vol. 27, No. 1A (Jan-Feb 2007), pp. 267-272 
Rath, K.S., Funk, H.M., Bowling, M.C., Richards, W.E., \& Drew, A.F. (2010). Expression of soluble interleukin-6 receptor in malignant ovarian tissue. American Journal of Obstetrics and Gynecology Vol. 203, No. 3 (September 2010), pp. 230.e1-8

Robinson-Smith, T.M., Isaacsohn, I., Mercer, C.A., Zhou, M., Van Rooijen, N., Husseinzadeh, N., McFarland-Mancini, M.M., \& Drew, A.F. (2007). Macrophages mediate inflammation-enhanced metastasis of ovarian tumors in mice. Cancer Research Vol 67, No. 12 (June 2007), pp. 5708-5716

Rosen, D.G., Mercado-Uribe, I., Yang, G., Bast, R.C. Jr, Amin, H.M., Lai, R., \& Liu, J. (2006). The role of constitutively active signal transducer and activator of transcription 3 in ovarian tumorigenesis and prognosis. Cancer Vol. 107, No. 11 (December 2006), pp. 2730-2740

Saydmohammed, M., Joseph, D., \& Syed V. (2010). Curcumin suppresses constitutive activation of STAT-3 by up-regulating protein inhibitor of activated STAT-3 (PIAS3) in ovarian and endometrial cancer cells. Journal of Cellular Biochemistry Vol. 110, No. 2 (May 2010), pp. 447-456

Scambia, G., Testa, U., Benedetti Panici, P., Foti, E., Martucci, R., Gadducci, A., Perillo, A., Facchini, V., Peschle, C., \& Mancuso, S. (1995). Prognostic significance of interleukin 6 serum levels in patients with ovarian cancer. British Journal of Cancer Vol. 71, No. 2 (February 1995), pp. 354-356

Scarlett, U.K., Cubillos-Ruiz, J.R., Nesbeth, Y.C., Martinez, D.G., Engle, X., Gewirtz, A.T., Ahonen, C.L., \& Conejo-Garcia, J.R. (2009). In situ stimulation of CD40 and Toll-like receptor 3 transforms ovarian cancer-infiltrating dendritic cells from immunosuppressive to immunostimulatory cells. Cancer Research Vol. 69, No. 18 (September 2009), pp. 7329-7337

Selvendiran, K., Bratasz, A., Kuppusamy, M.L., Tazi, M.F., Rivera, B.K., \& Kuppusamy, P. (2009). Hypoxia induces chemoresistance in ovarian cancer cells by activation of signal transducer and activator of transcription 3. International Journal of Cancer Vol. 125, No. 9 (November 2009), pp. 2198-2204

Selvendiran, K., Kuppusamy, M.L., Ahmed, S., Bratasz, A., Meenakshisundaram, G., Rivera, B.K., Khan, M., \& Kuppusamy, P. (2010). Oxygenation inhibits ovarian tumor growth by downregulating STAT3 and cyclin-D1 expressions. Cancer Biology $\mathcal{E}$ Therapy Vol. 10, No. 4 (August 2010), pp. 386-390

Seo, J.H., Jeong, K.J., Oh, W.J., Sul, H.J., Sohn, J.S., Kim, Y.K., Cho do, Y., Kang, J.K., Park, C.G., \& Lee, H,Y. (2010). Lysophosphatidic acid induces STAT3 phosphorylation and ovarian cancer cell motility: their inhibition by curcumin. Cancer Letters Vol. 288, No. 1 (February 2010), pp. 50-56

Smith, L.H. (2006). Early clinical detection of ovarian cancer: a review of the evidence. Expert Rev Anticancer Ther Vol. 6, No. 7 (July 2006), pp. 1045-1052

Stevinson, C., Steed, H., Faught, W., Tonkin, K., Vallance, J.K., Ladha, A.B., Schepansky, A., Capstick, V., \& Courneya, K.S. (2009). Physical activity in ovarian cancer survivors: associations with fatigue, sleep, and psychosocial functioning. Int J Gynecol Cancer Vol. 19, No. 1 (January 2009), pp. 73-78

Straub, R.H., Cutolo, M., Buttgereit, F., \& Pongratz, G. (2010). Energy regulation and neuroendocrine-immune control in chronic inflammatory diseases. Journal of Internal Medicine Vol. 267, No. 6 (June 2010), pp. 543-560 
Takeda, A., Toda, T., Shinohara, S., Mogi, Y., \& Matsui, N. Factors contributing to higher hematocrit levels in hemodialysis patients not receiving recombinant human erythropoietin. Am J Kidney Dis Vol. 40, No. 1 (July 2002), pp. 104-109

Thompson, M.S., \& Mok, S.C. (2009). Immunopathogenesis of ovarian cancer. Minerva Medica Vol. 100, No. 5 (October 2009), pp. 357-370

van Crevel, R., Karyadi, E., Netea, M.G., Verhoef, H., Nelwan, R.H., West, C.E., \& van der Meer, J.W. (2002) Decreased plasma leptin concentrations in tuberculosis patients are associated with wasting and inflammation. Journal of Clinical Endocrinology and Metabolism Vol 87, No. 2 (February 2002), pp. 758-763

van der Zee, A.G., de Cuyper, E.M., Limburg, P.C., de Bruijn, H.W., Hollema, H., Bijzet, J., Krans, M., \& de Vries, E,G. (1995). Higher levels of interleukin-6 in cystic fluids from patients with malignant versus benign ovarian tumors correlate with decreased hemoglobin levels and increased platelet counts. Cancer Vol. 75, No. 4 (February 1995), pp. 1004-1009

Visintin, I., Feng, Z., Longton, G., Ward, D.C., Alvero, A.B., Lai, Y., Tenthorey, J., Leiser, A., Flores-Saaib, R., Yu, H., Azori, M., Rutherford, T., Schwartz, P.E., \& Mor G. (2008). Diagnostic markers for early detection of ovarian cancer. Clinical Cancer Research Vol. 14, No. 4 (February 2008), pp. 1065-1072

Wang, Y., Niu, X.L., Qu, Y., Wu, J., Zhu, Y.Q., Sun, W.J., \& Li, L.Z. (2010). Autocrine production of interleukin-6 confers cisplatin and paclitaxel resistance in ovarian cancer cells. Cancer Letters Vol. 295, No. 1 (September 2010), pp. 110-123

Weinrib, A.Z., Sephton, S.E., Degeest, K., Penedo, F., Bender, D., Zimmerman, B., Kirschbaum, C., Sood, A.K., Lubaroff, D.M., \& Lutgendorf, S.K. (2010). Diurnal cortisol dysregulation, functional disability, and depression in women with ovarian cancer. Cancer Vol 116, No. 18 (September 2010), pp. 4410-4419

Wertel, I., Nowicka, A., Rogala, E., \& Kotarski, J. (2011). Peritoneal immune system in patients with advance epithelial ovarian cancer. International Reviews of Immunology Vol. 30, No. 2-3 (Apr-Jun 2011), pp. 87-101

Yang, J., Wang, Y., Gao, Y., Shao, J., Zhang, X.J., \& Yao, Z. (2009). Reciprocal regulation of 17beta-estradiol, interleukin-6 and interleukin-8 during growth and progression of epithelial ovarian cancer. Cytokine Vol. 46, No. 3 (June 2009), pp. 382-391

Yigit, R., Massuger, L.F., Figdor, C.G., \& Torensma, R. (2010). Ovarian cancer creates a suppressive microenvironment to escape immune elimination. Gynecologic Oncology Vol. 117, No. 2 (May 2010), pp. 366-372

Yigit, R., Figdor, C.G., Zusterzeel, P.L., Pots, J.M., Torensma, R., \& Massuger, L.F. (2011). Cytokine analysis as a tool to understand tumour-host interaction in ovarian cancer. European Journal of Cancer (April 2011)

Zhang, L., Conejo-Garcia, J.R., Katsaros, D., Gimotty, P.A., Massobrio, M., Regnani, G., Makrigiannakis, A., Gray, H., Schlienger, K., Liebman, M.N., Rubin, S.C., \& Coukos, G. (2003). Intratumoral T cells, recurrence, and survival in epithelial ovarian cancer. The New England Journal of Medicine Vol. 348, No. 3 (January 2003), pp. 203-213

Zhang, X., Liu, P., Zhang, B., Wang, A., \& Yang, M. (2010) Role of STAT3 decoy oligodeoxynucleotides on cell invasion and chemosensitivity in human epithelial ovarian cancer cells. Cancer Genetic and Cytogenetics Vol. 197, No. 1 (February 2010), pp. 46-53. 


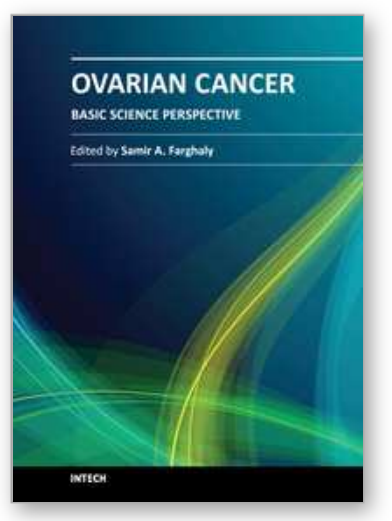

\author{
Ovarian Cancer - Basic Science Perspective \\ Edited by Dr. Samir Farghaly
}

ISBN 978-953-307-812-0

Hard cover, 406 pages

Publisher InTech

Published online 17, February, 2012

Published in print edition February, 2012

Worldwide, Ovarian carcinoma continues to be responsible for more deaths than all other gynecologic malignancies combined. International leaders in the field address the critical biologic and basic science issues relevant to the disease. The book details the molecular biological aspects of ovarian cancer. It provides molecular biology techniques of understanding this cancer. The techniques are designed to determine tumor genetics, expression, and protein function, and to elucidate the genetic mechanisms by which gene and immunotherapies may be perfected. It provides an analysis of current research into aspects of malignant transformation, growth control, and metastasis. A comprehensive spectrum of topics is covered providing up to date information on scientific discoveries and management considerations.

\title{
How to reference
}

In order to correctly reference this scholarly work, feel free to copy and paste the following:

Antonio Macciò and Clelia Madeddu (2012). Inflammation and Ovarian Cancer, Ovarian Cancer - Basic Science Perspective, Dr. Samir Farghaly (Ed.), ISBN: 978-953-307-812-0, InTech, Available from:

http://www.intechopen.com/books/ovarian-cancer-basic-science-perspective/inflammation-and-ovarian-cancer-

\section{INTECH}

open science | open minds

\section{InTech Europe}

University Campus STeP Ri

Slavka Krautzeka 83/A

51000 Rijeka, Croatia

Phone: +385 (51) 770447

Fax: +385 (51) 686166

www.intechopen.com

\section{InTech China}

Unit 405, Office Block, Hotel Equatorial Shanghai

No.65, Yan An Road (West), Shanghai, 200040, China 中国上海市延安西路65号上海国际贵都大饭店办公楼 405 单元

Phone: $+86-21-62489820$

Fax: +86-21-62489821 
(C) 2012 The Author(s). Licensee IntechOpen. This is an open access article distributed under the terms of the Creative Commons Attribution 3.0 License, which permits unrestricted use, distribution, and reproduction in any medium, provided the original work is properly cited. 